\title{
Upscaling of the permeability by multiscale wavelet transformations and simulation of multiphase flows in heterogeneous porous media
}

\author{
M. Reza Rasaei • Muhammad Sahimi
}

Received: 20 April 2008 / Accepted: 15 September 2008 / Published online: 24 October 2008

(C) The Author(s) 2008. This article is published with open access at Springerlink.com

\begin{abstract}
We describe a new approach for simulation of multiphase flows through heterogeneous porous media, such as oil reservoirs. The method, which is based on the wavelet transformation of the spatial distribution of the single-phase permeabilities, incorporates in the upscaled computational grid all the relevant data on the permeability, porosity, and other important properties of a porous medium at all the length scales. The upscaling method generates a nonuniform computational grid which preserves the resolved structure of the geological model in the near-well zones as well as in the high-permeability sectors and upscales the rest of the geological model. As such, the method is a multiscale one that preserves all the important information across all the relevant length scales. Using a robust front-detection method which eliminates the numerical dispersion by a high-order total variation diminishing method (suitable for the type of nonuniform upscaled grid that we generate), we obtain highly accurate results with a greatly reduced computational cost. The speedup in the computations is up to over three orders of
\end{abstract}

M. R. Rasaei · M. Sahimi $(\bowtie)$

Mork Family Department of Chemical Engineering and

Materials Science, University of Southern California,

Los Angeles, CA 90089-1211, USA

e-mail:moe@iran.usc.edu

M. R. Rasaei

Department of Chemical Engineering and Institute

of Petroleum Engineering, University of Tehran,

Tehran 11365-4563, Iran

e-mail:mrasaei@ut.ac.ir magnitude, depending on the degree of heterogeneity of the model. To demonstrate the accuracy and efficiency of our methods, five distinct models (including one with fractures) of heterogeneous porous media are considered, and two-phase flows in the models are studied, with and without the capillary pressure.

Keywords Two-phase flows • Geological model • Upscaling $\cdot$ Wavelet transformations

\section{Introduction}

Computer simulation of multiphase flows in heterogeneous and large-scale porous media, such as petroleum reservoirs, involves intensive computations (see, for example, [79]). Advances in measurement and estimation techniques, together with considerable progress in the development of theoretical and computational methods for characterization of heterogeneous porous media, provide significant and accurate amount of data on their important properties. Based on such data, modern geostatistical techniques [46] generate highly-resolved geological models of natural porous media. Due to computational limitations (mainly computation time), however, it is usually very difficult, if not impossible, to carry out simulation of multiphase flows using the geological model. It is, therefore, necessary to upscale the properties of the geological model's grid blocks in order to develop a coarsened grid that can be used in the computer simulation with an affordable amount of computation time while yielding accurate results. Moreover, the correct appraisal of the potential production of an oil reservoir must take into account the 
effect of the uncertainties in its properties. Production forecast made by a reservoir's stochastic model allows one to quantify the effect of the uncertainties on its performance by simulating multiphase flows in many realizations of the reservoir-a very time-consuming task. To reduce the simulation time, upscaling of the realizations of the reservoir's geological model is, perhaps, the only feasible remedy.

To simulate the flow of a single fluid in a reservoir, the most important parameter is the spatial distribution of the absolute permeability. This aspect of the problem is now well understood (see, for example, [57, 74, 99]). Various classification of the upscaling methods is possible based on the methods of their derivation (analytical or numerical), the dependence on the boundary conditions (local/extended-local, global/quasi-global, local/global), and the geological model employed (deterministic versus stochastic).

Analytical methods have the advantage that they are usually faster than numerical algorithms that are based on the numerical solution for the pressure distribution. They have the disadvantage that when applied to a sector outside their strict domain of validity, they quickly become inaccurate. Numerical methods are, therefore, more accurate but may become computationally too intensive.

In the local approaches, the upscaled transmissibilities for the faces of the upscaled blocks are computed using the generic flow solutions in the fine grid centered around the faces (see, e.g., $[22,69]$. Such methods have been refined by several groups $[40,100]$. The boundary conditions are typically of the Dirichlet type, or periodic, imposed on the fine grid. Local methods may produce unsatisfactory results if large-scale connected paths exist within a reservoir. To improve the accuracy, several approaches have been suggested, but they are significantly more expensive computationally. For example, [101] derived coarse-scale properties based on several global flows, while [41, 64] formulated the global upscaling as an optimization problem for a given flow scenario.

The local/global method was recently suggested by [14] in order to improve the upscaled representation of large-scale connectivities in a given geocellular grid. In this method, a standard (extended) local method is used to obtain the approximate upscaled permeability or transmissibility field. Then, a coarse global simulation is carried out. When the solution is interpolated for the local scales, the appropriate Dirichlet boundary condition is obtained for the next iteration. The iteration between the global and local scales is continued until the coarse field is converged. Chen and Durlofsky [13] developed an adaptive local/global upscaling procedure that provides coarse-scale parameters that are adapted for more general types of global flows (such as, for example, those driven by a well, or a particular set of boundary conditions). Gerritsen et al. [34] introduced a multilevel local/global method, which combines the local/global upscaling in order to improve the representation of the large-scale connectivities.

Simulation of multiphase flows, however, also involves adjustments to the flow of the various fluid phases through the connected blocks of the upscaled grid. To accomplish this task, many methods have been proposed, including upscaling techniques that employ the so-called pseudo-functions, the dual-mesh approach, and coarsening methods that generate a computational grid with nonuniform blocks. The pseudofunction technique is perhaps the most widely used method of upscaling of multiphase flows. It consists of replacing the original saturation-dependent relative permeabilities at the length scale over which the data are measured by fictitious functions that mimic the fluid flow process in the upscaled computational grid. Various versions and refinements of this basic concept have been proposed [19, 37, 43, 90]. Emmanuel and Cook [31] proposed pseudo-functions for well completions in an upscaled grid. The most widely used method of calculating the dynamic pseudo-functions was proposed by [49] who also introduced pseudo-capillary pressure functions. Despite its popularity, the KB method does not yield accurate results for strongly heterogeneous reservoirs; [48, 51] presented methodologies suitable for heterogeneous reservoirs that contain a hierarchy of relevant and distinct length scales. Stone [89] and others $[9,39]$ used the average total mobility in order to avoid computing the phase potentials in the upscaled grid that the $\mathrm{KB}$ method requires. Pickup and Sorbie [70] showed that one must characterize a heterogeneous reservoir by a tensorial effective relative permeability and, hence, tensorial pseudo-functions. A review of such concepts and techniques was given by [8] (see also [7]).

An accurate alternative to pseudo-functions, known as the dual-mesh (DM) method [33, 95], solves for the pressure distribution in the upscaled grid and includes the fine-scale heterogeneities in the computation of the phase saturations. Arbogast and Bryant [4] introduced a version of the DM method that upscales the transmissivities. They used a mixed finite-element (FE) method in which the fine-scale effects are localized by a boundary condition at the boundaries of the coarse blocks. The influence of the small-scale heterogeneities 
is then coupled with the coarse-scale effects using the appropriate Green functions. Audigane and Blunt [6] presented a 3D extension of the method using a finitedifference (FD) technique. The DM methods make no assumption regarding the relative significance of the viscous, capillary and gravitational forces, and generate negligible numerical dispersion, but their computational speed-up over the original geological model is hardly larger than 10 . Hou and $\mathrm{Wu}$ [42] employed a FE approach and constructed specific basis functions that capture the influence of the heterogeneities at small scales. The method was further refined by [15]. Lee et al. [52] developed a flux-continuous scheme for $2 \mathrm{D}$ models and extended the method [53] to 3D models. Further improvement of the method was suggested by [44]. More recently, [45, 56] proposed a multiscale finite-volume approach for computing the effective coarse-scale transmissibilities with tensorial permeabilities for 3D unstructured grids.

Generally speaking, although one can accurately compute the effective absolute permeabilities of the upscaled grid blocks by uniform coarsening, one also obtains an excessively smooth upscaled permeability distribution that does not honor the important effect of the distribution's tails at the fine scale, hence, leading to inaccurate predictions for such important quantities as the breakthrough time of the displacing fluid. Durlofsky et al. [23] introduced an upscaling method whereby a grid of finer resolution is used in the regions of high fluid velocities, but upscaled, homogenized description is utilized for the rest of the flow domain. Similar to the DM method, in Durlofsky et al.'s approach, the relative permeabilities are not upscaled. The fine-scale relative permeability functions are used for the upscaled grid blocks, hence, making the technique process-independent, a procedure consistent with the homogenization theory of multiphase flows at large scales [3, 10, 77]. Nonuniform grid coarsening offers significant computational speed-up and general applicability but at the cost of increasing numerical dispersion and decreasing accuracy for non-separable length scales.

Numerous grid selection methods rely on dynamic responses in order to identify the grid blocks in the geological model through which the fluids pass $[12,23$, $96,97]$. But, they are effective for accurately preserving the essential features of fluid flow at the fine scale only when the boundary conditions remain relatively constant. Because the pressure field in the near-well zones usually changes severely in the radial direction, such upscaling approaches are not usually accurate if they are used both in such zones and in the regions far from the wells. To address this problem, [24] developed an approach to calculate the transmissivities and well indices for single-phase flows based on the solution to the local well-driven flow.

We may also distinguish upscaling methods in terms of the stencils used to compute the flux across a block's face. In the two-point flux approximation (TPFA), the flux is computed based on the pressures in the two blocks that share the same face. In the more accurate multipoint flux approximation (MPFA), on the other hand, additional blocks are involved, which lead naturally to larger stencils than those in the TPFA methods. In 3D structured grids, for example, the MPFA method developed by [1] results in a 27-point stencil for the cellcentered pressure equations, compared with the sevenpoint stencils used in the TPFA methods. The approach has been studied by others as well $[30,53]$.

Aside from their high computational costs, the MPFA methods, when utilized in cases with strong permeability anisotropy, suffer from nonmonoticity. That is, the solution for the pressure distribution may contain unphysical oscillations [65]. The TPFA methods also suffer from a somewhat similar shortcoming in that they yield inconsistent approximate solutions to the singlephase pressure distribution when utilized in the same cases. Perhaps surprisingly though, the TPFA methods combined with the local/global procedures have been shown $[13,14]$ to provide accurate upscaled transmissibilities, when utilized in modeling flow in reservoirs with complex geologies. On the other hand, [50] presented a compact multipoint method that allows the MPFA stencil to vary spatially.

In this paper, we develop a new and highly efficient approach for simulation of multiphase flows based on the use of wavelet transformations. The method is a multiscale approach that acts as an "intelligent" grid generator at all the relevant length scales that are incorporated in the geological model. For the cases that we study, the method carries out the upscaling solely based on the spatial distribution of the single-phase permeabilities and yields very accurate results. The plan of this paper is as follows: in Section 2, we briefly describe important properties of wavelet transformations that we will use in our computations. Section 3 describes upscaling of the geological model based on wavelet transformation of the spatial distribution of the permeabilities, while in Section 4, we describe the geological model that we use in our work. The numerical technique that we develop and utilize to solve the governing flow equations is described in Section 5, after which the results are presented and discussed in Section 6. Section 7 discusses the speed-up in the 
computations that the wavelet-based method yields. The paper is then concluded with a discussion of the extension of the method to more complex models of porous media.

\section{Wavelet transformations}

We assume that the reservoir's heterogeneity is represented by the distribution $f[K(\mathbf{x})]$, where $K(\mathbf{x})$ is the single-phase permeability of a grid block of the reservoir's geological model with its center at $\mathbf{x} ; f(K)$ contains correlations at all the length scales that are incorporated in the geological model. Fourier transformation (FT) is inadequate for analyzing such data because all the important information about the data's localization properties will be lost in such an analysis. It is essential to represent such data in terms of localized functions. The requirement of locality was a prime motivation for developing the wavelet analysis and wavelet transformations (WT) since (see below) wavelet functions are nonzero over only limited intervals and, therefore, are localized.

In general, one must distinguish a continuous WT (CWT) from a discrete WT (DWT). A CWT is (similar to a continuous FT) used for analyzing and detecting unusual features of a data set. A DWT, on the other hand, is (similar to a discrete FT) used for data compression and reconstruction. A WT utilizes a function $\psi_{a b}(\mathbf{X})$, obtained by translating and rescaling of a mother wavelet $\psi(\mathbf{x})$, which is selected from a family of such functions:

$\psi_{a b}(\mathbf{X})=\frac{1}{a^{d / 2}} \psi[(\mathbf{x}-\mathbf{b}) / a]$,

where $a>0$ is the rescaling parameter, b represents translation of the wavelet, and $d$ is the system's dimensionality. As the word wavelet suggests, $\psi(\mathbf{x})$ has a wavelike behavior within a limited domain. More generally, each of the coordinates $\mathbf{x}=(x, y, z)$ may have its own distinct rescaling parameter, $a=\left(a_{x}, a_{y}, a_{z}\right)$, in which case $a^{d / 2}$ is replaced by $\sqrt{a_{x} a_{y} a_{z}}$. The choice of $a^{-d / 2}$ in Eq. 1 is not essential (particularly for the problem studied in this paper). In fact, a normalization factor $a^{-1}$ is often used in 1D which has the advantage of giving more weight to the small scales. A wavelet is usually required to have a zero mean:

$$
\int_{-\infty}^{\infty} \psi(\mathbf{x}) d \mathbf{x}=0
$$

More generally, $\psi(\mathbf{x})$ is often required to have a number of vanishing moments:

$$
\begin{aligned}
& \int_{-\infty}^{\infty} \int_{-\infty}^{\infty} \int_{-\infty}^{\infty} x^{\alpha} y^{\beta} z^{\gamma} \psi(x, y, z) d x d y d z=0, \\
& 0 \leq \alpha+\beta+\gamma \leq M
\end{aligned}
$$

which improves the efficiency of the wavelet at detecting singularities or unusual features of a dataset. The possibility of selecting the wavelet from a family of such functions affords one great flexibility in choosing a suitable wavelet for analyzing a given dataset.

The WT of $K(\mathbf{x})$, also called its wavelet detail coefficient, is then defined by

$$
\begin{aligned}
D(a, \mathbf{b}) & =\int_{-\infty}^{\infty} K(\mathbf{x}) \psi_{a b}(\mathbf{x}) d \mathbf{x} \\
& =\frac{1}{a^{d / 2}} \int_{-\infty}^{\infty} K(\mathbf{x}) \psi[(\mathbf{x}-\mathbf{b}) / a] d \mathbf{x}
\end{aligned}
$$

Equation 4 exhibits a fundamental property of a wavelet analysis: by computing the WT of $K(\mathbf{x})$, using a shifted and rescaled wavelet, one analyzes $K(\mathbf{x})$ at increasingly coarser $(a>1)$ or finer $(a<1)$ length scales. Therefore, by devising a proper scheme based on the DWT of $K(\mathbf{x})$, one can, at any length scale of interest, systematically compress information on $K(\mathbf{x})$ in those parts of the computational grid (representing the geological model) where detailed description is not needed and obtain a new grid with larger blocks. The precise manner by which the compression is done is described below. The transformation $K(\mathbf{x}) \rightarrow D(a, \mathbf{b})$ may be inverted exactly, yielding a reconstruction formula (which is important to the problem that we consider in this paper; see below):

$K(\mathbf{x})=C_{\psi}^{-1} \int d \mathbf{b} \int \frac{1}{a^{d+1}} \psi_{a b}(\mathbf{x}) D(a, \mathbf{b}) d a$,

in which $C_{\psi}$ is a normalization constant, and the domains of the integrals represent all possible values of $a$ and $\mathbf{b}$. Equation 5 implies that a WT decomposes a data set as a linear superposition of the wavelets $\psi_{a b}$ with coefficients $D(a, \mathbf{b})$, and that the natural measure in the parameter space $(a, \mathbf{b})$ is $d \mathbf{b} d a / a^{d+1}$ which is invariant not only under scale translation but also under dilation.

Since in the upscaling problem, we utilize a computational grid, a DWT is used which is obtained by restricting the parameters $a$ and $\mathbf{b}$ to the grid points. Hence, in 1D for example, one writes,

$D_{j, k}=2^{-j / 2} \int \psi\left(2^{-j} x-k\right) K(x) d x$,

where $j$ and $k$ are integers. One can construct functions $\psi$ for which the set $\left\{\psi_{j, k}, j, k\right\}$ is an orthonormal basis 
of in the real space. Such a basis has all the important and useful properties of the wavelets, including space localization, and yields fast computational algorithms. The construction of the basis uses the fact that almost all the wavelets' orthonormal bases are derived from a multiresolution analysis, which we now describe briefly.

$D_{j, k 1, k 2}$ contains information only about the difference or contrast between two approximations of the same function at two successive length scales. The most accurate approximation of a function at a fixed scale is given by another function called the scaling function $\phi(\mathbf{x})$ such that, for example in $1 \mathrm{D}$, the set $\left\{\phi_{j, k} \equiv \phi\left(2^{j} x-k\right)\right\}$ is orthonormal, with $k$ being an integer and the definition of $\phi_{j, k}(\mathbf{x})$ being similar to that of $\psi_{j, k}(\mathbf{x})$. Whereas the mean value of $\psi(\mathbf{x})$ over the entire space is zero, the mean value of $\phi(\mathbf{x})$ is unity over the same space. In analogy with $D_{j, k 1, k 2}$, the wavelet approximate or scale coefficients are defined by

$S_{j, k}=\int_{-\infty}^{\infty} \phi_{j, k}(\mathbf{x}) K(\mathbf{x}) d \mathbf{x}$,

As described below, the scale and wavelet functions are interrelated.

Consider, first, the 1D wavelets. The orthonormal wavelets $\psi$ are constructed based on the two-scale (also known as the scaling or refining) equation:

$\phi(x)=\sqrt{2} \sum_{n=-\infty}^{\infty} h_{n} \phi(2 x-n), h=\langle\phi 1, n \mid \phi\rangle$

from which the orthonornal wavelets are generated by

$\psi(x)=\sqrt{2} \sum_{n=-\infty}^{\infty}(-1)^{n-1} h_{-n-1} \phi(2 x-n)$,

where the $h_{n}$ are usually called the filter coefficients. One important family of orthonormal wavelets with compact support, i.e., those that are nonzero over only small intervals of $x$, is the Daubechies set of wavelets of order $M[20,21]$ (usually referred to as DBM), used in this paper. They possess the property that their first $M$ moments are zero. The scaling function $\phi(x)$ is related to those at the finer length scales by an equation similar to Eq. 8:

$\phi(x)=\sqrt{2} \sum_{n=0}^{L-1} h_{n} \phi(2 x-n)$,

where $L=2 M$ and, similar to Eq. 9 ,

$\psi(x)=\sqrt{2} \sum_{n=0}^{L-1} m_{n} \phi(2 x-n)$.
The quantities $m_{n}$, which are also filter coefficients, are related to $h_{n}$ by (compare Eqs. 9 and 11) $m_{n}=$ $(-1)^{n} h_{L-n-1}$, with $n=0,1, \ldots, L-1$. They are usually nonzero for only a few values of $n$ since the wavelets have compact support.

The simplest wavelet is the Haar or DB1 wavelet which, in $1 \mathrm{D}$, is defined by

$\psi_{0,1}(x)=\left\{\begin{array}{cl}1 & 0 \leq x<1 / 2 \\ -1 & 1 / 2 \leq x<1 \\ 0 & \text { otherwise }\end{array}\right.$

The filter coefficients of the Haar wavelet are $\left(h_{0}, h_{1}\right)=$ $(1 / \sqrt{2})(1,1)$ and, therefore, $\left(m_{0}, m_{1}\right)=(1 / \sqrt{2})(-1,1)=$ $\left(-h_{1}, h_{0}\right)$. The factor $1 / \sqrt{2}$ (often not included) is to ensure orthonormality between $\phi$ and $\psi$. Press et al. [71] provided an extensive list of the filter coefficients for a variety of wavelets.

Using the Haar wavelet, we demonstrate a key property of WTs. It is not difficult to see that the Haar wavelet expresses the approximation to a function $f$ by wavelets by replacing an adjacent pair of steps by one wider step and one wavelet. The wider step measures the average of the initial pair of steps, while the wavelet, formed by two alternating steps, measures the difference between the initial pair of steps. Thus, the sum of two adjacent steps with one half width produces the basic unit step function, $\phi_{0,1}=\phi_{0,1 / 2}+\phi_{1 / 2,1}$. Similarly, the difference of the two steps yields the corresponding Haar wavelet, $\psi_{0,1}=\phi_{0,1 / 2}-\phi_{1 / 2,1}$. The decomposition is a fundamental property of a WT: a WT decomposes a function $K(\mathbf{x})$ into two separate components or functions-a scaling function $\phi(\mathbf{x})$ and a wavelet $\psi(\mathbf{x})$-both acting on $K(\mathbf{x})$. The two functions separate the components into averages and differences, with a "wavelength" equal to the "window" over which $\phi$ and $\psi$ are nonzero. Moreover, a WT is recursive so that it can be applied in succession to any set of averages produced using the wavelets to generate other levels of averages and details, and so on. The averages computed by a WT are not, in general, the arithmetic averages but depend on the wavelet function used. Only the Haar wavelet produces the arithmetic average. These properties are the key to the application of a multiresolution WT to the problem studied in this paper.

Two-dimensional wavelets are usually built in separable form by tensor products of the 1D wavelets (sometimes referred to as the Cohen-Daubechies wavelets). Therefore, in $2 \mathrm{D}$, one has one scaling function:

$\phi_{j, k_{1}, k_{2}}(x, y)=\phi_{j, k_{1}}(x) \phi_{j, k_{2}}(y)$, 
and three wavelet functions:

$\psi_{j, k_{1}, k_{2}}^{(1)}(x, y)=\phi_{j, k_{1}}(x) \psi_{j, k_{2}}(y)$,

$\psi_{j, k_{1}, k_{2}}^{(2)}(x, y)=\psi_{j, k_{1}}(x) \phi_{j, k_{2}}(y)$,

$\psi_{j, k_{1}, k_{2}}^{(3)}(x, y)=\psi_{j, k_{1}}(x) \psi_{j, k_{2}}(y)$,

where the superscripts 1,2 , and 3 indicate, respectively, the correspondence of the wavelets with the horizontal, vertical, and diagonal changes in the data. The extension to $3 \mathrm{D}$ wavelets is straightforward: One has seven detail and one scale coefficients. All the results reported in this paper were obtained using the Daubechies-four (DB4) wavelet. The choice was made based on the efficiency of the computations and accuracy of the results. Using higher-order wavelet does not only improve the accuracy but also increases the computations' time. The filter coefficients $\left(h_{0}, h_{1}, h_{2}, h_{3}\right)$ of the DB4 wavelets are $\frac{1}{8}[(\sqrt{2}+\sqrt{6}),(3 \sqrt{2}+\sqrt{6})$, $(3 \sqrt{2}-\sqrt{6}),(\sqrt{2}-\sqrt{3})]$.

\section{Wavelet scale up of the single-phase permeabilities}

One major goal of this paper is to show that in many cases, the wavelet-based upscaling that uses only singlephase flow properties is accurate and efficient for many models of highly heterogeneous reservoirs. Therefore, we upscale only the single-phase permeabilities of the grid blocks in the geological model.

While, undoubtedly, there may be cases for which some multiphase flow properties might need to be upscaled, for the cases that we study in the present paper, as well as the three-dimensional model reservoir that we have studied elsewhere [73], namely, the SPE-10 model [16], there seem to be no need for upscaling of any multiphase properties.

The wavelet upscaling of the single-phase permeabilities was developed in our previous papers [26, 60, 82] which, for completeness, is briefly described here. We consider 2D models of oil reservoirs (or other types of porous media); its extension to $3 \mathrm{D}$ which poses no conceptual difficulty over what we describe here. The method has been applied elsewhere [73] to upscaling of the SPE-10 model, geological model of an oil reservoir in which the single-phase permeabilities vary over eight orders of magnitude. The geological model is represented by a square grid of equal-size blocks, which may be obtained by discretizing the governing equations by the standard five-point FD approximation or from a finite-element discretization of the governing equations
[60]. The dimensions of the grid blocks depend on the resolution of the distribution $f(K)$. Each square block is assigned a permeability $K$ selected from the distribution $f[K(\mathbf{x})]$ (the extension to the case in which each block is characterized by a permeability tensor is straightforward; see below), taking into account the correlation between the blocks' permeabilities.

To begin the upscaling, we first compute the DWT of $K(\mathbf{x})$. Associated with the DWT at every block with its center at $\mathbf{x}=\left(k_{1}, k_{2}\right)$ [at $\mathbf{x}=\left(k_{1}, k_{2}, k_{3}\right)$ in 3D] are four wavelet coefficients (eight in 3D). Following Eqs. 6 and 7 , the four wavelet coefficients are, in more precise forms, given by

$$
\begin{aligned}
& S_{l, k_{1}, k_{2}}=\int_{\Omega} K(\mathbf{x}) \phi_{l, k_{1}, k_{2}}(\mathbf{x}) d \mathbf{x}, \\
& D_{l, k_{1}, k_{2}}^{(\ell)}=\int_{\Omega} K(\mathbf{x}) \psi_{l, k_{1}, k_{2}}^{(\ell)}(\mathbf{x}) d \mathbf{x},
\end{aligned}
$$

where $l$ is the level of upscaling $(l=0$ represents the geological model), and $\Omega$ is the domain of the problem. As mentioned above, $S_{l, k 1, k 2}$ contains information about the (weighted) average permeability at $\mathbf{x}$ at a fixed scale of the grid, while the detail coefficients $D_{l, k_{1}, k_{2}}^{(\ell)}$ measure the contrast or difference between $K(\mathbf{x})$ of a block with its center at $\mathbf{x}$ in the coarser scale and those of its neighbors in the immediate finer scale (one with blocks half the size of the coarser scale), with $\ell=1,2$, and 3 (in $3 \mathrm{D}, \ell=1, \mathrm{~L}, 7)$ measuring the contrasts in $K$ between the blocks in the $x, y$, and the diagonal directions, respectively. Thus, a large $S_{l, k 1, k 2}$ indicates that the permeability at $\mathbf{x}=\left(k_{1}, k_{2}\right)$ is large, while large values of the detail coefficients indicate large contrasts between the permeabilities of two neighboring blocks in two successive scales. Accurate and efficient computations then require that we identify such zones of the grid, and upscale the rest of the grid, as their representation at the coarser scale suffices for taking into account their influence on the fluid flow process.

To make the identification, two thresholds, $\varepsilon_{s}$ and $\varepsilon_{d}$, are introduced $[26,60]$ and are set as a fraction of their corresponding largest values throughout the grid. The scale coefficient of each block is then examined. If it is larger than $\varepsilon_{s}$ (implying that the block's permeability is large), it is left intact, and the next grid block is examined. If, however, the examined scale coefficient is smaller than $\varepsilon_{s}$, the associated detail coefficients are examined and set to zero if they are smaller than their threshold $\varepsilon_{d}$. Setting $D_{l, k_{1}, k_{2}}^{(\ell)}$ means that the neighbor of the block centered at $\left(k_{1}, k_{2}\right)$ and in the direction $\ell$, which in the finer-scale grid is only one block (or one diagonal block) away from it, is not needed and, 
therefore, the two blocks merge to form a block twice as large. Sweeping the grid once and examining all the scale and detail coefficients result in the merger of many grid blocks, hence, generating a new grid with fewer blocks. The blocks' sizes in the new grid are no longer equal, as some of the blocks in the fine-scale grid have merged, while others have remained intact.

As mentioned earlier, one important property of a WT is its recursive nature. That is, one can repeatedly apply the transformation to a partially compressed set of data to obtain another more compressed set. Taking advantage of this property, the new grid is upscaled again by applying the DWT to the scale coefficients, computed at the previous step, which contain information about the permeabilities in the current (partially) upscaled grid, and calculating a new set of four coefficients for each block of the (partially) upscaled grid. The grid is then swept again to merge some of the blocks and form larger blocks. Typically, after a few sweeps (levels of upscaling $l$ ) the grid can no longer be coarsened efficiently. In practice, the number of upscaling levels depend on the broadness and structure (with or without correlation) of $f(K)$.

It also depends on the nature of the problem. The reduction in the number of the blocks depends on the thresholds $\varepsilon_{s}$ and $\varepsilon_{d}$. Clearly, the higher the two thresholds, the larger is the number of the grid blocks that are merged to form larger upscaled blocks and, hence, the smaller is the number of grid points that are utilized in the flow simulations. Instead of the permeability $K(\mathbf{x})$, a WT can be applied to any other geological or petrophysical property that one may wish to base the upscaling on. For example, one may upscale the grid based on the distribution of the local flow velocities in single-phase flow through the model. Moreover, the structure of the grid around the (injection and/or production) wells is important. Thus, we may consider two upscaling scenarios: in one, the geological model is upscaled based only on the wells' positions, implying that the blocks are upscaled uniformly in the regions far from wells, and the fine grid structure in the nearwell regions is maintained (or coarsened less). In the second scenario, we consider both the wells' positions and the permeabilities in the upscaling process. Some of the upscaling methods developed in the past also consider the position of the wells in the grid.

After generating the upscaled blocks, an important issue is computing their effective permeabilities, which can be done by many methods. One method is based on the analogy between fluid flow and electrical currents. For example, a configuration that consists of four neighboring square blocks that make a larger square block, each having its own permeability, is replaced, after upscaling by a larger block with an effective permeability $K^{\prime}$ given by the following general form [67]:

$K^{\prime}=K^{\prime}\left(K_{i}, K_{i j}, h_{i}, h_{j}\right)$

where $K_{i}$ is the permeability of block $i$ with, $i=1-4$, $K_{i j}=K_{i}+K_{j}$, and $h_{i}$ and $h_{j}$ are the linear sizes of the blocks $i$ and $j$. The appearance of the blocks' linear sizes $h_{i}$ and $h_{j}$ is necessitated by the rescaling of the smaller blocks into a larger block. Since the first level of upscaling involves the equal-size blocks of the initial fine grid, all the linear sizes $h_{i}$ and $h_{j}$ are equal and cancel out. But, the upscaling beyond the first level involves unequal-size grid blocks, which necessitates introduction of $h_{i}$ and $h_{j}$.

If the permeabilities are direction-dependent, then the transformation (Eq. 19) is used once for each direction in order to compute the equivalent directiondependent permeabilities. The second method is based on reconstruction of $f(K)$, i.e., computing its inverse WT after some of the scale and detail coefficients have been set to zero. The effective permeabilities of the upscaled blocks are then assigned based on reconstructed $f(K)$. We used both methods and found that the difference between the two is negligible.

In general, starting with the geological model, the final upscaled grid will contain a distribution of block sizes, as a large number of the blocks in the geological model merge at various levels of upscaling to form larger blocks. Then, blocks of unequal sizes may become neighbors (see below). Such blocks (and the grid points that represent them) have only three (or fewer) neighbors (in 2D); therefore, the governing equations for fluid flow cannot, at such points, be discretized using the standard FD approximation. To overcome this difficulty, we use the finite-volume method which is best suited for use in computational grids that contain unequal blocks $[38,82]$. We will come back to this point shortly.

Applications of the WT to problems related to petroleum reservoirs and other types of porous media have recently been increasing. In addition to analyzing the seismic data which were one of the prime motivations for developing the WT, the applications also include numerical simulation of flow and transport equations [62], upscaling of a conductivity or permeability field $[26,60,82]$, pressure transient analysis [5, 25, 28, 47, 87], upscaling of miscible displacements in heterogeneous porous media [27], reservoir characterization [11, 55, $59,63,66]$, identification of the fracture density along wells in oil and gas reservoirs [81], as well as problems involving turbulent transport and chemical reactions in the atmosphere [38]; for a recent review, see [78, 80]. 
We point out that [17] also attempted to use the WT for upscaling using a method different from what we describe here. In addition, in their method, the grid was upscaled uniformly throughout (that is, the upscaled blocks all have the same size). As such, the method is not much different from many of the previous upscaling methods that did not use the WT.

\section{The geological model}

To put the method under a stringent test, we use a synthetic geological model in which not only is the distribution $f(K)$ of the local permeabilities relatively broad (typically distributed over three orders of magnitude) but also contains long-range correlations. To this end, we generate the permeabilities by the fractional Brownian motion (FBM), a stochastic process that induces long-range correlations in the permeabilities. The extent of the correlations that are generated by the FBM is as large as the system's linear size. Briefly, the FBM is characterized by a spectral density given by

$S(\omega)=\frac{a(d)}{\left(\sum_{i} \omega_{i}^{2}\right)^{\alpha}}$,

where $a(d)$ is $d$-dependent constant, and $\alpha=H+d / 2$, with $d$ being the dimensionality of the system. The Hurst exponent $H$ is such that $H>1 / 2(<1 / 2)$ implies positive (negative) correlations, while for $H=1 / 2$ the successive increments in the FBM-generated values are uncorrelated. There is considerable evidence that the FBM-type correlations are ubiquitous in oil reservoirs and groundwater aquifers (see, for example, [61]). To generate a permeability distribution for a stratified reservoir, we rewrite the $2 \mathrm{D}$ power spectrum of the FBM as,

$S(\omega)=\frac{a(d)}{\left(a_{1} \omega_{x}^{2}+\omega_{y}^{2}\right)^{\alpha}}$,

where $a_{1}$ is a numerical constant such that with $a_{1} \neq 1$, one obtains stratifications that are more or less parallel to the $x$-direction. Extension of Eq. 21 to 3D is straightforward. We emphasize that the upscaling method described in this paper is independent of the permeability distribution $f(K)$.

To provide a simple example, consider a $1 \mathrm{D}$ system with 16 blocks of different permeabilities generated by a 1D FBM. The synthetic sequence of the $K$ values (level $l=0)$ is given by, $(0.60,0.916,0.845,0.60,0.605$, $0.996,1.0,0.91,0.83,0.463,0.29,0.054,0.048,0.217$, $0.0628,0.0257)$, so that the largest and smallest $K$ differ by a factor of about 40 . After one level of coarsening $(l=1)$, using $\varepsilon_{s}=\varepsilon_{d}=0.9$ one obtains,

$(0.758,0.727,0.605,0.996,1.00,0.91,0.83,0.463,0.17$,

$$
0.13,0.044) \text {, }
$$

hence, reducing the system to an eleven-block system. The $K$ values for the coarsened blocks were calculated by reconstruction, i.e., by computing the inverse DWT of the distribution of $K$, after setting to zero the appropriate wavelet scale and detail coefficients. Note, for example, that the first two blocks on the left side (with $K=0.60$ and 0.916 ) merged, as did the third and fourth blocks, while blocks number 5-10 remained intact. The second level of upscaling $(l=2)$ yields,

(0.742, 0.605, 0.996, 1.00, 0.91, 0.83, 0.463, 0.17, 0.088),

which is a nine-block system. The upscaling can continue further until it reduces the system to 2-3 blocks.

It might seem from the foregoing simple example that a direct upscaling algorithm based on the blocks' permeabilities and their contrast with those of their neighbors may result in the same coarsened grid. However, that is not the case. It is only in the wavelet space that the differences between the blocks' permeabilities, and their absolute values can be used as a criterion for upscaling. This is because, as is well-known in the theory of WTs, a function may have a small value at a given point but have large wavelet scale and detail coefficients in the wavelet space and vice versa. This is similar to image processing with the wavelets which is done by digitizing the image, computing its WT, and compressing it by a method similar to what we described above: the compression is not done in the real space but in the wavelet space. Otherwise, the compressed image will be distorted.

\section{Numerical simulation of two-phase flows}

We simulate a water-flooding process as an example of a typical two-phase flow in a heterogeneous porous medium, such as an oil reservoir, and solve the problem in both the geological and upscaled models in order to assess the accuracy of the upscaling method. Combining the Darcy's law and the mass conservation equation and ignoring the effect of gravity yield the following equations for two-phase flow of oil and water in a reservoir:

$$
\begin{aligned}
& B_{\mathrm{o}}^{-1} \nabla \cdot\left(K \lambda_{\mathrm{o}} \nabla \Phi_{\mathrm{o}}\right)-\frac{q_{\mathrm{o}}}{\rho_{\mathrm{o}}}=\frac{\partial}{\partial t}\left(\frac{\varphi S_{\mathrm{o}}}{B_{\mathrm{o}}}\right), \\
& B_{\mathrm{w}}^{-1} \nabla \cdot\left(K \lambda_{\mathrm{w}} \nabla \Phi_{\mathrm{w}}\right)-\frac{q_{\mathrm{w}}}{\rho_{\mathrm{w}}}=\frac{\partial}{\partial t}\left(\frac{\varphi S_{\mathrm{w}}}{B_{\mathrm{w}}}\right),
\end{aligned}
$$


where, $\Phi_{\mathrm{w}}=P_{\mathrm{o}}-P_{\mathrm{c}}$, with $P_{\mathrm{c}}=P_{\mathrm{o}}-P_{\mathrm{w}}, P_{\mathrm{o}}$ and $P_{\mathrm{w}}$ being the pressures in the oil and water phases, $\lambda_{0}$ and $\lambda_{\mathrm{w}}$ are, respectively, the mobility of the oil and water, and $\left(B_{\mathrm{o}}, q_{\mathrm{o}}\right)$ and $\left(B_{\mathrm{w}}, q_{\mathrm{w}}\right)$ are their corresponding formation volume factor and flow rate, respectively. The densities $\rho_{\mathrm{o}}$ and $\rho_{\mathrm{w}}$ are evaluated under the standard conditions. We then combine Eqs. 22 and 23 to obtain a single equation for $P_{\mathrm{o}}$, containing no explicit time derivatives of the phase saturations:

$$
\begin{aligned}
& B_{o}\left[\nabla \cdot\left(K \frac{\lambda_{\mathrm{o}}}{B_{\mathrm{o}}} \nabla P_{\mathrm{o}}\right)-\frac{q_{\mathrm{o}}}{\rho_{\mathrm{o}}}\right] \\
& \quad+B_{\mathrm{w}}\left[\nabla \cdot\left(K \frac{\lambda_{\mathrm{w}}}{B_{\mathrm{w}}}\left(\nabla P_{\mathrm{o}}-\nabla P_{c}\right)\right)-\frac{q_{\mathrm{w}}}{\rho_{\mathrm{w}}}\right]=\varphi C \frac{\partial P_{\mathrm{o}}}{\partial t}
\end{aligned}
$$

Where $C_{\mathrm{i}}=-B_{\mathrm{i}}^{-1} \partial B_{\mathrm{i}} / \partial P_{\mathrm{i}}(\mathrm{i}=o, w)$ represents the phase compressibility, $C_{\mathrm{r}}=-\varphi_{\mathrm{i}}^{-1} \partial \varphi / \partial P_{\mathrm{o}}$ is the rock's compressibility, and $C=C_{\mathrm{r}}+C_{\mathrm{o}} S_{\mathrm{o}}+C_{\mathrm{w}} S_{\mathrm{w}}$.

The governing equations for $P_{\mathrm{o}}$ and $S_{\mathrm{o}}$ are solved by a finite-volume (FV) method, which is most suitable for the type of computational grid that we generate by the upscaling method. Although most of the current commercial simulators for reservoir simulation utilize FD approximation to the governing flow equations, due to its great flexibility in handling a computational grid with unequal blocks, use of the FV method is also becoming increasingly popular. In fact, the commercial simulator FLUENT (used for computational fluid dynamics) utilizes the FV method.

To utilize the FV method, we first integrate both sides of Eq. 24 over a control surface $A$ (control volume in $3 \mathrm{D})$, to obtain

$$
\begin{aligned}
& \iint C \varphi \frac{\partial P_{\mathrm{o}}}{\partial t} d A \\
&=\int\left\{B_{\mathrm{o}}\left[\nabla \cdot\left(K \frac{\lambda_{\mathrm{o}}}{B_{\mathrm{o}}} \nabla P_{\mathrm{o}}\right)-\frac{q_{\mathrm{o}}}{\rho_{\mathrm{o}}}\right]\right. \\
&\left.\quad+B_{\mathrm{w}}\left[\nabla \cdot\left(K \frac{\lambda_{\mathrm{w}}}{B_{\mathrm{w}}}\left(\nabla P_{\mathrm{o}}-\nabla P_{c}\right)\right)-\frac{q_{\mathrm{w}}}{\rho_{\mathrm{w}}}\right]\right\} d A
\end{aligned}
$$

The left side of Eq. 25 is evaluated by assuming that the oil pressure in any block can be represented by an average value. Likewise, the flow rates $q_{\mathrm{o}}$ and $q_{\mathrm{w}}$ are assumed to represent the average rates in any gridblock. The time derivative is then approximated by a suitable FD form. Therefore, the left side of Eq. 25 is written, for example, as, $C \varphi \Delta S\left(P_{\mathrm{i}}^{n+1}-P_{\mathrm{i}}^{n}\right) / \Delta t$, if we use a forward FD approximation, where $\Delta S$ is the surface of the block, $P_{\mathrm{i}}^{n}$ is the pressure after $n$ time steps at grid point $i$ representing a block, and $\Delta t$ is the time step. One, then, invokes the divergence theorem to convert the surface integrals on the right side of Eq. 25 to line integrals. Since the upscaled grid contains blocks of various sizes, all the terms on the right side of Eq. 25 after their conversion to line integrals via the divergence theorem must be evaluated carefully. Consider the configuration of the blocks shown in Fig. 1. For the western side of the base block $b$ we write the line integral as,

$$
\begin{aligned}
\int_{\mathrm{w}} K \frac{\lambda_{\mathrm{o}}}{B_{\mathrm{o}}} \nabla P_{\mathrm{o}} d y= & \sum_{j=1}^{N}\left(\frac{\ell_{j}+\ell_{b}}{\ell_{j} / K_{j}+\ell_{b} / K_{b}}\right)\left(\frac{\lambda_{\mathrm{o}, \text { up }}}{B_{\mathrm{o}}}\right)_{\mathrm{w} n}^{m} \\
& \times\left(\frac{P_{j}-P_{o}}{\ell_{j}+\ell_{b}}\right) S_{b j}
\end{aligned}
$$

Here, $S_{b j}$ is the common surface (side) between two adjacent blocks $j$ and $b, \ell_{j}$ and $\ell_{b}$ are, respectively, the cell-to-face distances for the neighbor cell $j$ and the base cell $b ; N \geq 1$ is the number of neighboring blocks on the western side of the base cell $b(N=1$ if the two blocks have the same size $),\left(P_{j}, K_{j}\right)$ and $\left(P_{b}, K_{b}\right)$ are the average pressures and permeabilities representing blocks $j$ and $b$, and $\lambda_{\mathrm{o}, u p}$ is the mobility of the oil phase in the front block. The superscript $m$ is the time step number at which the quantities are evaluated.

The discretization represented by Eq. 26 results in a local leading truncation error on the order of $O(1 / h)$, when applied to $\tilde{h}$ adaptive nonuniform grids $[32,72]$. The analysis of [29] indicated that in grids with large aspect ratios the truncation error depends on the grid interface and aspect ratios, the permeabilities, and the pressure gradient acting tangentially on the grid interface (between two unequal blocks). Thus, in order to eliminate such errors (or reduce them to very small values), we followed the approach suggested by [29]. Consider, for example, the configuration of the blocks

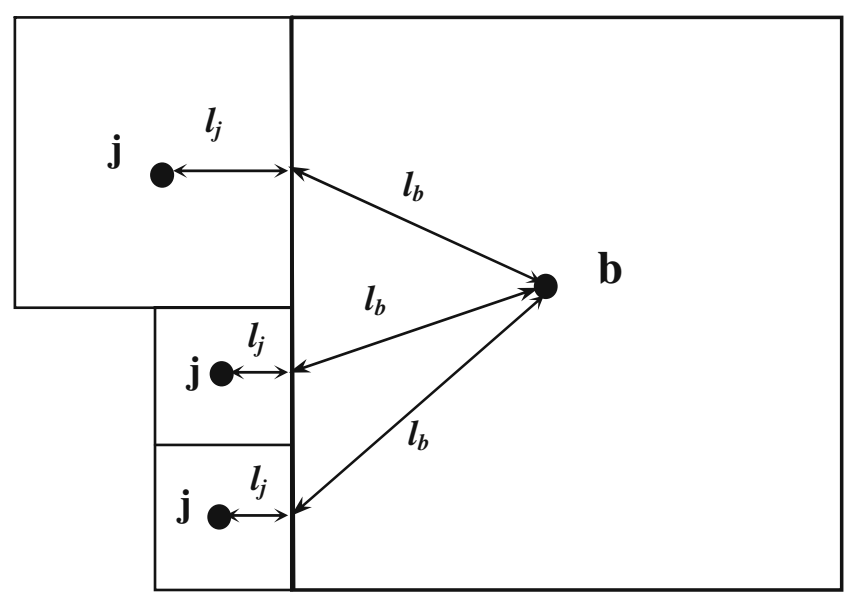

Fig. 1 A base block $b$ and its "western" neighbors $j$ in the finitevolume formulation 


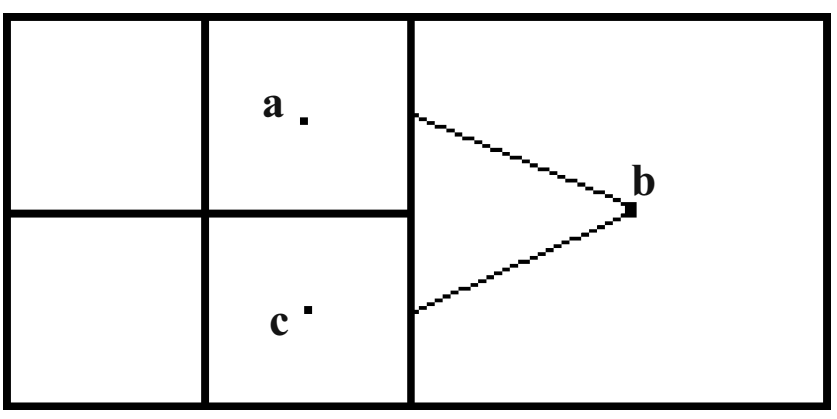

Fig. 2 Three blocks of unequal sizes used for computing the fluxes between them (see Eqs. 27-29)

shown in Fig. 2. To calculate the flux between $a$ and $c$ in the figure, we use the standard expression based on the harmonic averaging of the blocks' permeabilities:

$J_{a c}=2 \frac{h_{x}}{h_{y}} \frac{K_{a} K_{c}}{K_{a}+K_{c}}\left(P_{a}-P_{c}\right)$,

whereas to compute, for example, the flux between $a$ and $b$ we use,

$J_{a c}=4 \frac{h_{x}}{h_{y}} \frac{K_{a} K_{b} K_{c}}{4 K_{a} K_{b}+K_{a} K_{b}+K_{b} K_{c}}\left(P_{b}-\left(P_{a}+P_{c}\right) / 2\right)$,

with a similar expression for $J_{a b}$, where $h_{x}$ and $h_{y}$ are the blocks' linear dimensions at the finer scale. In an upscaling method which is not based on the wavelet function, Eq. 28 can be problematic. For example, if the permeability of either block $a$ or $c$ is very small, then the flux across both faces $a b$ and $c b$ will be very small or zero. More generally, if the faces between block $b$, block $a$, and block $c$ are of equal size, then the flux out of block $b$ is evenly distributed between the faces, which may lead to nonphysical oscillations in the pressure field. In the absence of the wavelet upscaling, an accurate way of addressing the issue is by the use of the so-called $L$ method [2].

However, the wavelet-based method proposed here automatically takes into account such effects. Sudden changes in the blocks' sizes and properties are correctly addressed by using the DB4 (or higher-order) wavelet functions, which consider extra blocks around the central block under examination for upscaling. The sudden changes will be reflected in the corresponding wavelet scale and detail coefficients, which may then lead to no upscaling at all of the central blocks and its merger with its neighbors.

In any event, Eq. 26 is used only when equal-size blocks are neighbors $(N=1)$, but for the case in which unequal blocks (with an interface ratio of 2) are neighbors, Eq. 26 is modified to

$$
\begin{array}{rl}
\int_{\mathrm{w}} & K \frac{\lambda_{\mathrm{o}}}{B_{\mathrm{o}}} \nabla P_{\mathrm{o}} d S \\
= & \sum_{j=1}^{N} 4\left(\frac{K_{a} K_{b} K_{c}}{4 K_{a} K_{b}+K_{a} K_{b}+K_{b} K_{c}}\right) \\
& \times\left(\frac{\lambda_{\text {o, up }}}{B_{\mathrm{o}}}\right)\left(\frac{S_{b j}}{l_{b}}\right)\left(P_{b}-\left(P_{a}+P_{c}\right) / 2\right),
\end{array}
$$

As described by [29], the extension to grids of larger interface ratios is straightforward.

The simulator first solves the governing equations for the pressure $P_{\mathrm{o}}$ of the oil phase and then computes the oil saturation $S_{\mathrm{o}}$ by solving Eq. 22. To obtain the solution, we use a combination of the implicit-pressure, explicit-saturation (IMPES) and the fully implicit methods. The limits $m=n$ and $m=n+1$ (where $n$ is the current time step number) of Eqs. 26 or 29 correspond, respectively, to the IMPES and fully implicit procedures. Expressions similar to Eqs. 26 or 29 are also written down for the eastern, northern, and southern neighbors of the base block $b$, and the resulting terms are all added up to represent the corresponding integral.

The IMPES method is conditionally stable and converges to the correct solution if the time step $\Delta t$ is selected carefully. If $\Delta t$ is too large, the numerical simulator may generate saturations that are larger than one. This possibility was eliminated by imposing the condition that the saturation and pressure changes in any grid block between two consecutive time steps must remain tightly bounded, which was achieved by adjusting $\Delta t$ such that the saturation changes between $t$ and $t+\Delta t$ in any grid block were no more than 0.05 , and the pressure changes no more than 100 psi. In addition, to guarantee the stability of the solution, an adaptive timestep method was used which automatically increased $\Delta t$ whenever the variables changed slowly and vice versa.

The advantage of the IMPES method is that, when used appropriately, it yields accurate results at a minimum computational cost. Its limitations are that, (a) because a large portion of the reservoir far from the wells may experience very slow changes in $P_{\mathrm{o}}$ and $S_{\mathrm{o}}$, it is not efficient to use very small $\Delta t$, and (b) it is not accurate if large variations in the dependent variables occur rapidly. The time-truncation errors are generally larger in implicit simulators [85, 93]. A fully implicit method provides the required stability but at a considerably higher computational cost.

The largest variations in $S_{\mathrm{o}}$ and $P_{\mathrm{o}}$ occur in the nearwell regions, which also control the maximum allowed 
time step $\Delta t$ when the flood front is close to them. Therefore, at the beginning of the injection, $\Delta t$ must be small, but when the front is no longer near the injection well, it can be much larger. When the front reaches the vicinity of the production well(s), $\Delta t$ must again be small. Therefore, we consider a small zone of grid blocks (of size $7 \times 7$ ) around each well in which we use the fully implicit procedure for discretizing the governing equations, while the IMPES method is used in the remaining part of the grid.

For the fully implicit part, we guess the $S_{\mathrm{o}}$ distribution at time $t$ and solve the discretized equations for $P_{\mathrm{o}}$. The $S_{\mathrm{o}}$ distribution is then computed based on the newly calculated $P_{\mathrm{o}}$ distribution. The procedure is iterated several times (typically about eight) until converged solutions are obtained. For the IMPES part, after computing the $P_{\mathrm{o}}$ distribution after each time step, we solve Eq. 22 in which the time-derivative term is discretized explicitly. In both cases, the discretized equations are solved by a combination of the NewtonRaphson and biconjugate-gradient methods.

To obtain accurate solutions for $P_{\mathrm{o}}$ and $S_{\mathrm{o}}$, one must improve the approximations that are made for estimating the upstream flow functions, which requires accurate evaluations of the phase mobilities. Harten and coworkers [35, 36, 103] introduced a method for evaluating the phase mobilities, usually referred to as the total variation diminishing (TVD) method, which is free of the instabilities.

It is increasingly being used in reservoir simulation $[76,98,102]$, especially for simulating miscible displacements where numerical dispersion may completely dominate the physical dispersion [58, 68, 84, 86, 92]. The method requires an appropriate front detection technique which can be based on the calculation of successive mobility ratios in the neighboring blocks. Higher-order TVD methods are constructed by introducing a variable mobility at each block face given by

$\lambda_{\text {o,face }}=\lambda_{\mathrm{o}, \text { up }}+\frac{1}{2} \eta_{1+1 / 2}\left(\lambda_{\mathrm{o}, \text { down }}-\lambda_{\mathrm{o}, \text { up }}\right)$,

where $\eta_{i+1 / 2}$ is called the mobility limiter. Here, $\lambda_{\mathrm{o} \text {, face }}=\lambda_{i+1 / 2}, \lambda_{\mathrm{o} \text {, up }}=\lambda_{\mathrm{i}}$, and $\lambda_{\mathrm{o}, \text { down }}=\lambda_{i+1}$. Moreover,

$r_{i+1 / 2}=\frac{\lambda_{\mathrm{o}, \text { up }}-\lambda_{\mathrm{o}, \text { up }-1}}{\lambda_{\mathrm{o}, \mathrm{down}}-\lambda_{\mathrm{o}, \mathrm{up}}}$.

Here, $\lambda_{\text {o,up-1 }}$ denotes the oil mobility of up-side block, attached to the upstream block of the base cell. $r_{i+1 / 2}$ varies in such a way that the scheme is, at least spatially, second order. The results obtained by using Eq. 30 are usually free of spurious oscillations. Several expressions for $\eta_{i+1 / 2}$ have been developed [75, 91, 94]. One of the most accurate limiters [94] is given by,

$\eta_{i+1 / 2}=\max \left\{\min \left[2 r_{i+1 / 2}, \frac{1}{2}\left(r_{i+1 / 2}+1\right), 2\right], 0\right\}$.

The above TVD method is applicable to FD-based simulations that use uniform grids. According to Eq. 32, in addition to the upstream and downstream mobilities, second-neighbor mobilities $\lambda_{\text {o, up }-1}$ enter the formulation as well. Since in a nonuniform grid, the base block might have several second-neighbor upstream blocks, we use the maximum mobility among such blocks as the representative of the upside mobility; that is, $\lambda_{\text {o,up }-1}=$ $\lambda_{i-1, \max }$. Such a choice guarantees that the approaching front will be detected by at least one of the neighboring blocks of the base block.

Let us point out that due to its multiscale nature of the wavelets, no assumption needs to be made regarding the significance, or lack thereof, of the capillary pressure. Clearly, since one obtains a grid with various block sizes, the significance of $P_{\mathrm{c}}$ is automatically diminishes in the large ones.

\section{Results and discussion}

We have carried out extensive simulations of water flooding in systems with various geometries and permeability fields. All the computations were carried out using a single $3.2 \mathrm{GHz}$ Pentium IV processor. What follows are the description of the model reservoirs and the discussions of the results.

Case 1 We consider a 2D waterflood in a quarter of a five-spot system in which the wells are located diagonally at the opposite corners of the grid. The geological model is represented by a $512 \times 512$ square grid. The permeability field is isotropic and is generated by a FBM with $H=0.2$, which corresponds to a heterogeneous field with negative correlations (large permeabilities are neighbors to small ones, and vice versa). The semivariogram of a FBM is given by

$$
\gamma(r)=\gamma_{1} r^{2 H}
$$

where $r$ is the lag, and $\gamma_{1}=\gamma(r=1)$. Equation 33 indicates that the semivariogram of a FBM should continue to increase as the lag $r$ increases (i.e., $\gamma(r)$ does not exhibit any sill), since the correlation length is as large as the system's size, and no cutoff scale for the correlations was introduced in the FBM. The minimum and maximum block permeabilities, $\left(K_{\min }\right.$, 
$\left.K_{\max }\right)$, are $(1 \mathrm{mD}, 1,620 \mathrm{mD})$ with a mean of about $735 \mathrm{mD}$ (the standard deviation of $\log K$ is about 1.0). The porosity was assumed to be constant everywhere. Figure 3 presents the upscaled permeability map, together with the corresponding grid where the smallest blocks represent the geological model's resolution. The upscaled grid was generated with the thresholds $\varepsilon_{s}=\varepsilon_{d}=0.8$. The number of grid blocks was reduced from the initial 262,144 to only 2,464 , with low coarsening levels in the high-permeability zones, high coarsening levels in the low-permeability regions, and no upscaling at all around the wells (see above).

Water is injected at one corner of the grid at a constant pressure of $6,000 \mathrm{psi}$, and the fluid is produced at constant total flow rate of $50 \mathrm{bbl} /$ day at the opposite corner. The relative permeability model used is a conventional Corey-type function,

$k_{r j}\left(S_{j}\right)=k_{0, j}\left(\frac{S_{j}-S_{r j}}{S_{j, \max }-S_{r j}}\right)^{n_{j}}$
Fig. 3 Case 1 upscaled permeability map and the corresponding computational grid. The smallest blocks represent the resolution of the original geological model
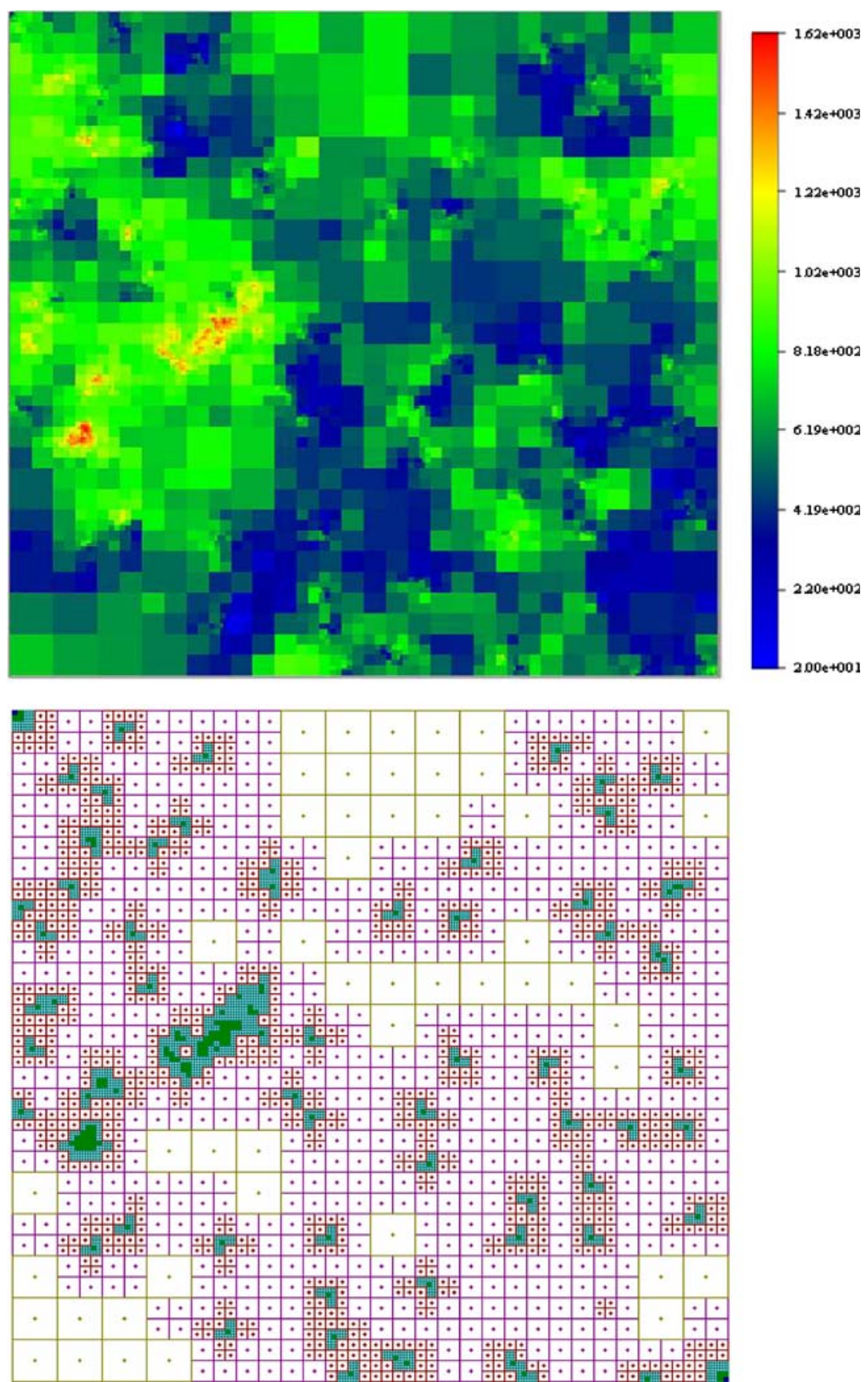
with $K_{0, \mathrm{o}}=K_{0, \mathrm{w}}=1, S_{\mathrm{o}, \max }=S_{\mathrm{w}, \max }=0.8, S_{\mathrm{ro}}=S_{\mathrm{rw}}=$ $0.2, n_{\mathrm{w}}=1.5$, and $n_{\mathrm{o}}=2$. The oil and water viscosities are taken, respectively, to be 5 and $1 \mathrm{cP}$, and gravity and capillary pressure effects are ignored. Moreover, we took, $C_{\mathrm{w}}=3 \times 10^{-6}$ and $C_{\mathrm{o}}=4 \times 10^{-6}$, both in $p s i^{-1}$. We emphasize that it neither poses any particular difficulty, nor does it increase the method's computational cost to use many different expressions for $K_{r j}\left(S_{j}\right)$ for different sectors of the grid. Figure 4 compares the $S_{\mathrm{w}}$ distributions 175 days after water injection into the reservoir, computed using both the geological and upscaled models. All the main features of the flood pattern in the geological model are captured by the upscaled grid. However, due to coarsening, the advancing finger becomes smoother in the upscaled model but causing only a very small delay in the breakthrough time in the production well. The delay effect can be seen in Fig. 5 where we compare the water flow rates at both wells in both the geological and upscaled models. These results indicate that, in the upscaled grid, the time at which the rate of water production has risen from zero has a small delay compared to that of the geological model. Despite this, the trends in the water flow rate curves of the two models are in very good agreement.

Since the regions around the wells retain their resolved structures in the upscaled model, and a fully implicit model is used in such regions to solve the governing equations (the IMPES formulation is utilized in the rest of the reservoir), we may expect close
Fig. 4 Distribution of water saturation after 175 days in a the geological model and b in the upscaled model
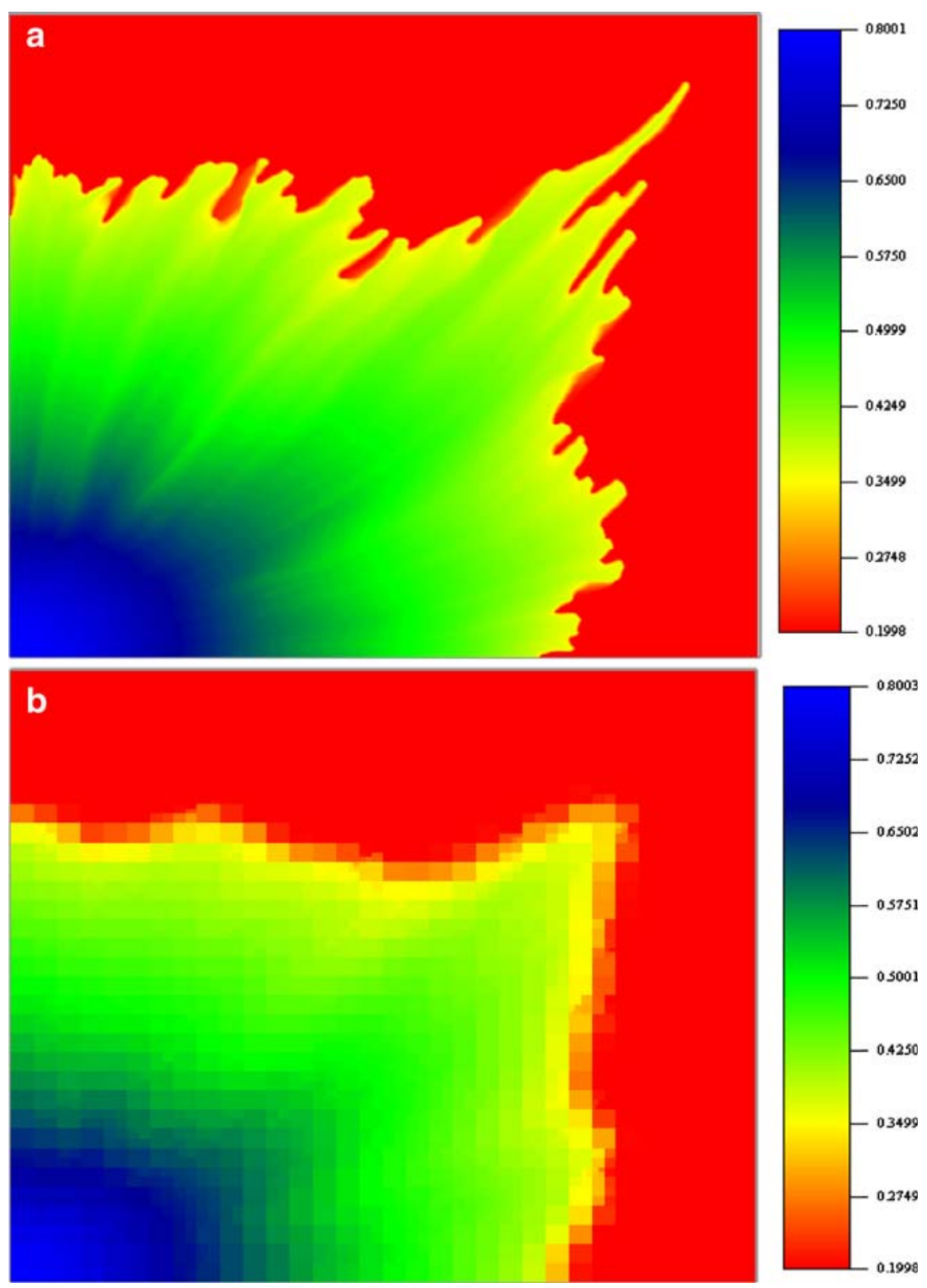


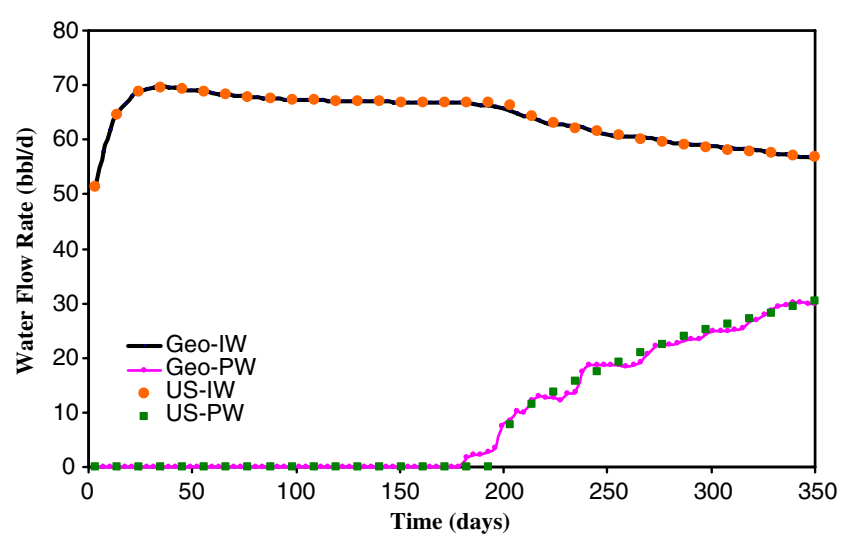

Fig. 5 Comparison of the water flow rates at the injection $(I)$ and production $(P)$ wells, using the geological $(\mathrm{Geo})$ and the upscaled (US) models

agreement between the two sets of results in terms of the bottom-hole pressures. Figure 6 confirms this expectation, where we show the computed well pressures using both the geological and the upscaled models. The small difference between the two computed pressures at the production well is related to the corresponding difference between the breakthrough times of the two models; the difference is about $2 \%$.

Case 2 In this case, the permeability field was stratified, with the layers being more or less parallel to the macroscopic flow, generated by an FBM with $H=0.2$ (and setting $a_{1} \neq 1$ in Eq. 21). The statistics of the permeability fields are similar to the first case (except that, due to stratification, the field is anisotropic), as is the size of the grid representing the geological model. Uniform porosity was assumed everywhere. In one case, the grid was upscaled using

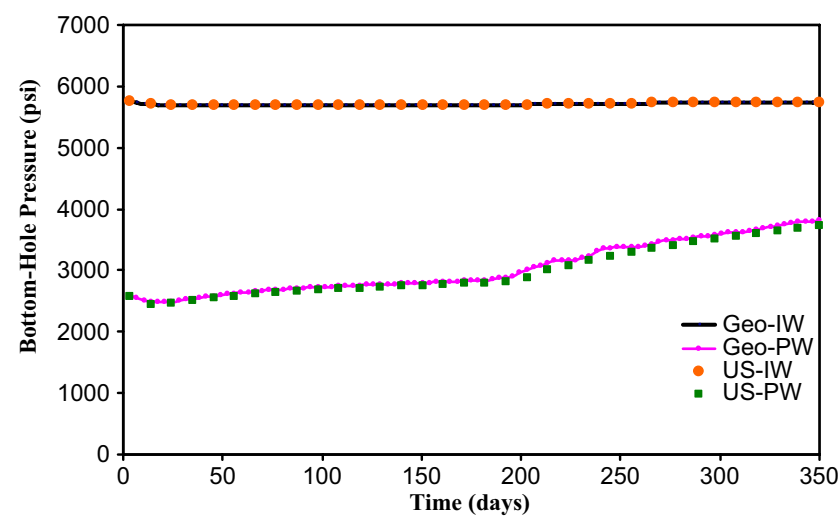

Fig. 6 Comparison of the bottom-hole pressures in the geological and upscaled models. The notations are the same as in Fig. 5 $\varepsilon_{s}=\varepsilon_{d}=1.0$, which corresponds to uniform coarsening of the grid. For the second case, $\varepsilon_{s}=\varepsilon_{d}=0.8$ were utilized. Moreover, different levels of upscaling (the parameter $l$ in Eqs. 17 and 18) were also utilized in order to assess their effect. The upscaled permeability map and the corresponding grid for $\varepsilon_{s}=\varepsilon_{d}=$ 0.8 and three coarsening levels $(l=3)$ are shown in Fig. 7. Note, in particular, the fine structure of the computational grid at the interfaces between neighboring layers as well as in the sectors with high permeabilities.

We simulated a water-flooding process in which water was injected into the reservoir on the left side of the grid, at all the grid blocks, at a constant pressure of $6,500 \mathrm{psi}$, and oil and water were produced from the opposite side at a constant pressure of 5,000 psi (equal to the initial pressure). No-flow boundary condition was imposed in the lateral direction. To impose severe condition on the simulations, we simulated a viscosity ratio, $M=100$. Similar to Case 1 , we neglected the capillary pressure. Figure 8 compares the water flow rates at the outlet face, computed both in the geological and upscaled models. We simulated the process in several different grids in which the blocks near the inlet and outlet of the system were upscaled at several different levels, ranging from zero (that is, the blocks were not upscaled) to one and two levels of upscaling (that is, one or two sweepings of the grid; see Section 3). As can be seen, very little difference between all the figures can be discerned. In addition, the values of the two thresholds and whether or not one upscales the grid blocks near the system's inlet and outlet appear to make very little difference. The absence of any effect of the resolution of grid blocks near the boundaries is due to the absence of the capillary pressure, making the end effects unimportant. We come back to this point shortly.

Figure 9 compares the computed water saturations $S_{\mathrm{w}}(x, y)$ after 400 days in the geological and upscaled models. Once again, the agreement between the two figures is excellent. Figure 10 compares the timedependence of the average reservoir pressures in the geological and upscaled models with three different levels of coarsening. All the important features of the curves agree quantitatively. Figure 11 makes the same type of comparison, but for the injected flow rates of water. Once again, all the cases agree completely with each other. Figure 12 compares the flow rates of the produced water in the geological model and those in the upscaled grids, one uniformly coarsened with $\varepsilon_{s}=\varepsilon_{d}=$ 1.0 and one level $(l=1)$ of upscaling (which reduces 
Fig. 7 Case 2 upscaled permeability map and the corresponding computational grid. The smallest blocks represent the resolution of the original geological model
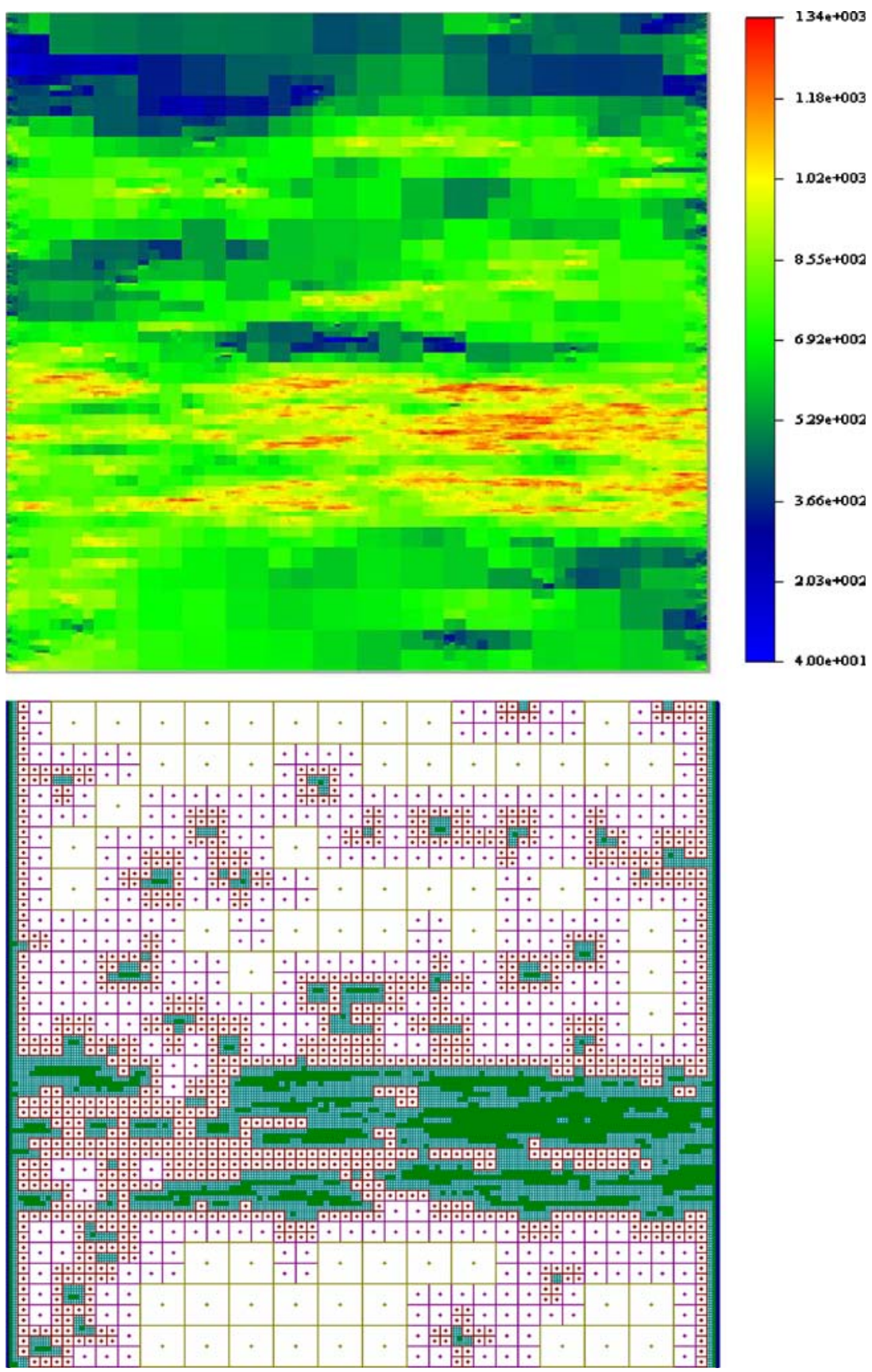

the number of the grid blocks by more than $98 \%$ ) and a second one with three levels of upscaling and $\varepsilon_{s}=$ $\varepsilon_{d}=0.8$. Once again, the agreement is excellent.

Case 3 This case was similar to Case 2, except that the capillary pressure was not neglected, and was represented by

$$
P_{\mathrm{c}}=\sigma \sqrt{\frac{\varphi}{K}}\left(\frac{S_{\mathrm{w}}-S_{\mathrm{w} i}}{1-S_{\mathrm{w} i}-S_{\mathrm{o}}}\right)^{4},
$$

with $\sigma=20 \mathrm{mN} / \mathrm{m}$ being the interfacial tension, $\varphi=0.3$ the porosity (assumed to be constant everywhere, although it can vary from block to block, if need be), and $S_{\mathrm{w} i}$ and $S_{\mathrm{or}}$ the irreducible water saturation and oil residual saturation. Note, however, that any capillary pressure curve, as well as different $P_{\mathrm{c}}$ curves for different zones of the grid, may be used. Let us also point out that the shape of the capillary pressure, which depends on the permeability $K$, has a strong effect on the saturation distribution and the computational stiffness 
Fig. 8 Comparison of the water flow rates in the anisotropic model produced at the outlet (right) face of the system if $P_{\mathrm{c}}$ is neglected. The results are for a the geological model and $\left(\varepsilon_{d}, \varepsilon_{s} l\right)=\mathbf{b}(1.0,1.0,2)$ c $(1.0,1.0,1) ; \mathbf{d}(1.0,1.0,0)$; e $(0.8,0.8,2)$, and $\mathbf{f}(0.8,0.8,1)$, where $l$ is the level of upscaling of the boundary nodes. Note that $\varepsilon_{s}=\varepsilon_{d}=1.0$ corresponds to uniform coarsening of the grid
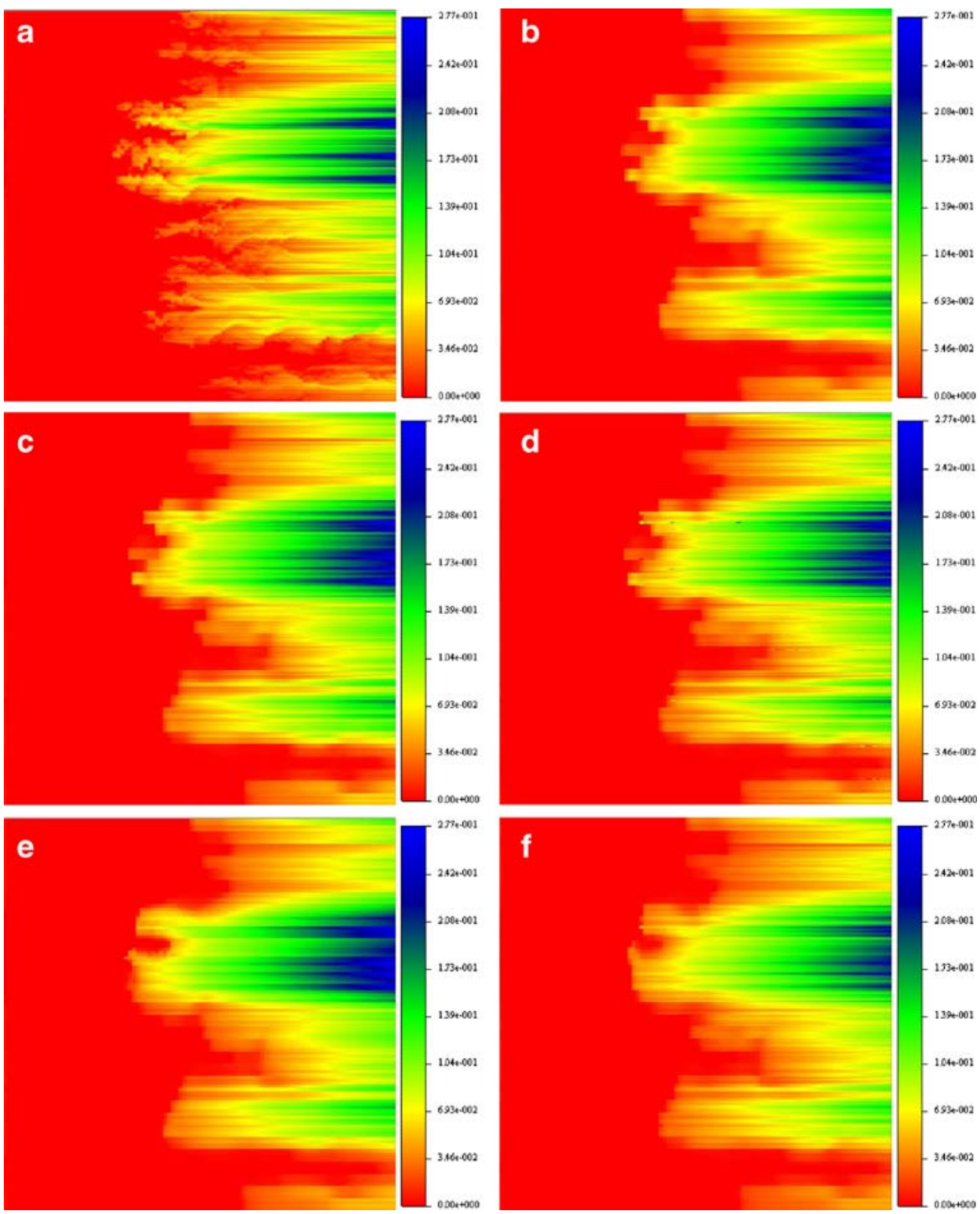

of the governing equations that we solve in order to compute the distribution. In particular, a vanishing, or a mildly varying, capillary pressure does not give rise to end effects that are usually seen in the simulations of two-phase flow with a strong capillary pressure (see below). Moreover, due to the capillary discontinuity, capillary end-effects appear at the system's inlet and outlet boundaries, giving rise to larger $S_{\mathrm{w}}$ in these regions than in the rest of the grid. To capture this effect accurately, the size of the blocks adjacent to the grid's left- and right-most boundaries can be held at the level of the geological model.

Figure 13 compares the local water flow rates at the outlet in the geological model with those computed in the upscaled models for two values of the thresholds $\varepsilon_{d}$ and $\varepsilon_{s}$ and one or two coarsening levels $l$.

To understand the results, we must consider the variations of $P_{\mathrm{c}}$ with the permeability $K$. In the geological model the permeability varies from about 1 to $1,340 \mathrm{mD}$. Thus, according to Eq. $35, P_{\mathrm{c}} \leq 1,200$ psi. In general, large capillary pressures tend to imbibe water into the low-permeability regions and smoothen the front. However, upscaling the blocks reduces the capillary forces in two important ways:

(1) The higher the blocks' permeabilities and the saturation contrasts between adjacent blocks, the higher is the capillary force between them. 

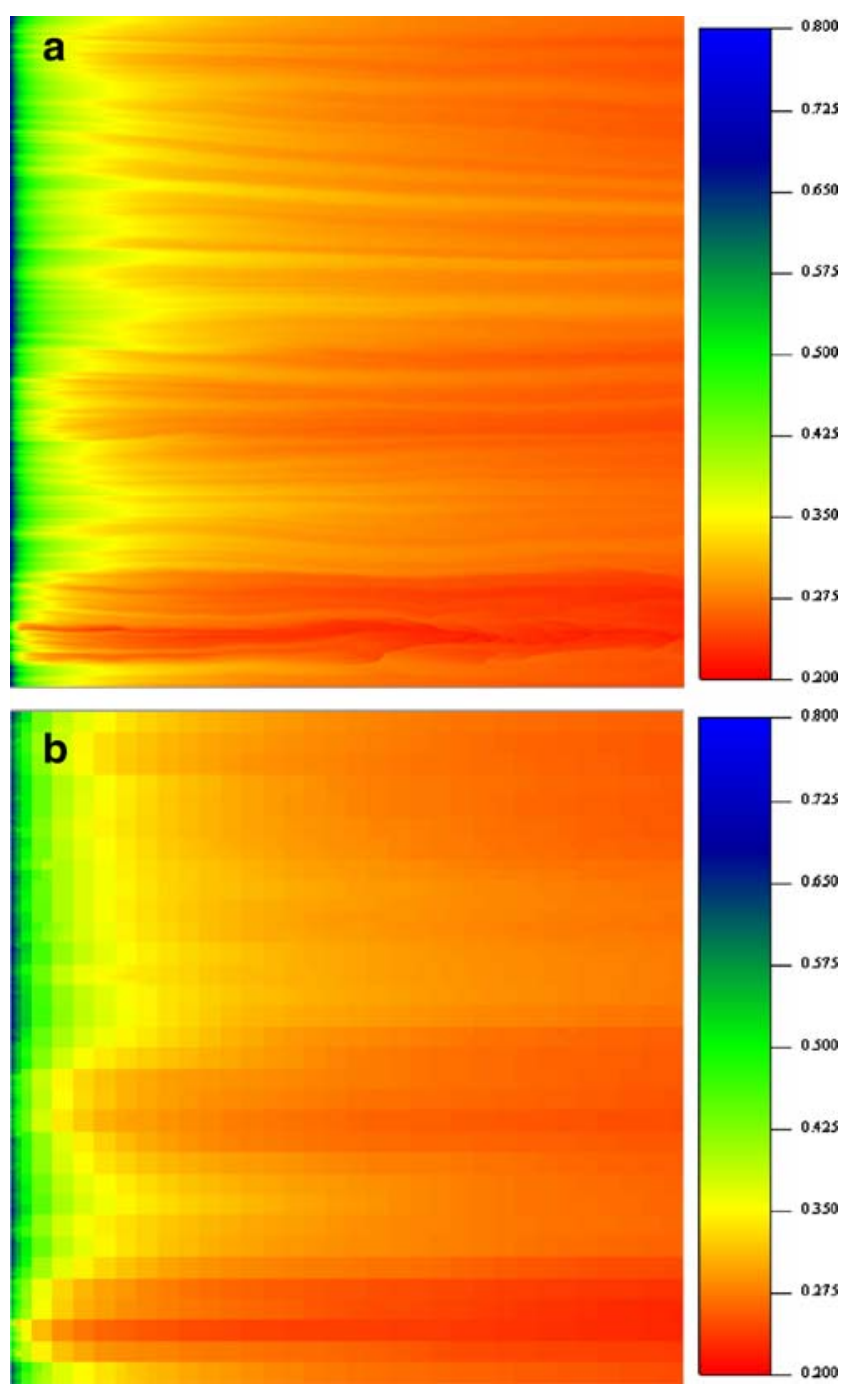

Fig. 9 Waterssaturation distribution after 400 days, if $P_{\mathrm{c}}$ is neglected, in the a anisotropic geological model and b upscaled model

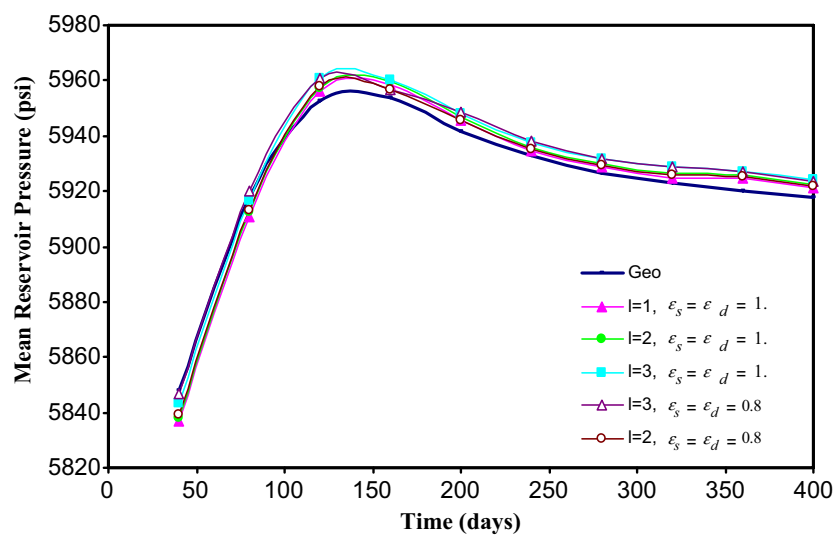

Fig. 10 Time-dependence of the mean reservoir pressure in the anisotropic geological (Geo) and several upscaled models. Capillary pressure was neglected

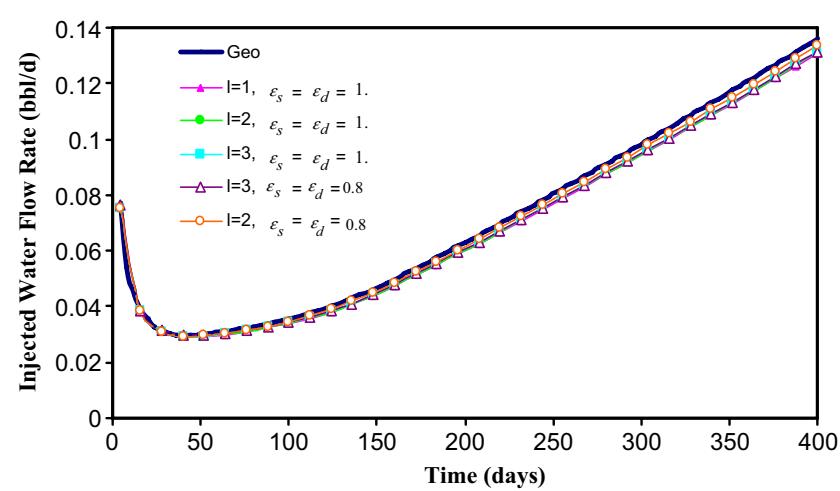

Fig. 11 Time-dependence of the injected water flow rates in the anisotropic geological ( $\mathrm{Geo})$ and upscaled models. Capillary pressure was neglected

Upscaling, however, smoothens both the permeability and saturation contrasts between the adjacent blocks and, hence, reduces the capillary forces between them.

(2) The capillary forces are inversely proportional to the blocks' sizes, implying that one, two, and three levels of upscaling reduce, respectively, the capillary forces by factors of $1 / 2,1 / 4$, and $1 / 8$. The net effect is the weakening of the role of capillary forces in smoothening the saturation profile. Consequently, one has a far more heterogeneous saturation distribution and water production history, as the grid is increasingly upscaled (see Fig. 8).

Such expectations are confirmed by the results shown in Fig. 13 and their comparison with those shown in Fig. 8. Figure 13d presents the case in which the blocks in a thin region near the left- and right-most

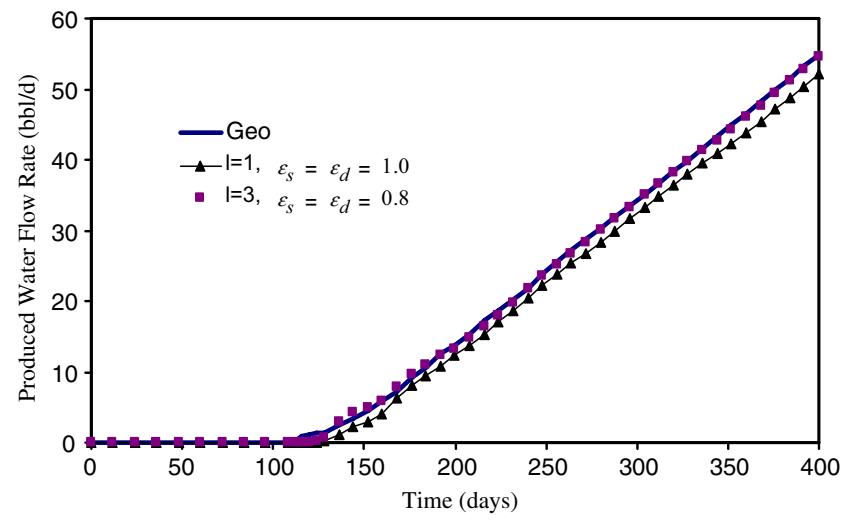

Fig. 12 Water flow rates in the anisotropic geological (Geo) model and in the upscaled grids, one uniformly coarsened $\left(\varepsilon_{s}=\right.$ $\left.\varepsilon_{d}=1.0\right)$ with one level of upscaling $(l=1)$ and a second one with three levels of upscaling. Capillary pressure was neglected 
Fig. 13 a-f Comparison of water flow rates for Case 3 model, with the capillary pressure, given by Eq. 35, included in the simulations. The notations are the same as those in Fig. 8
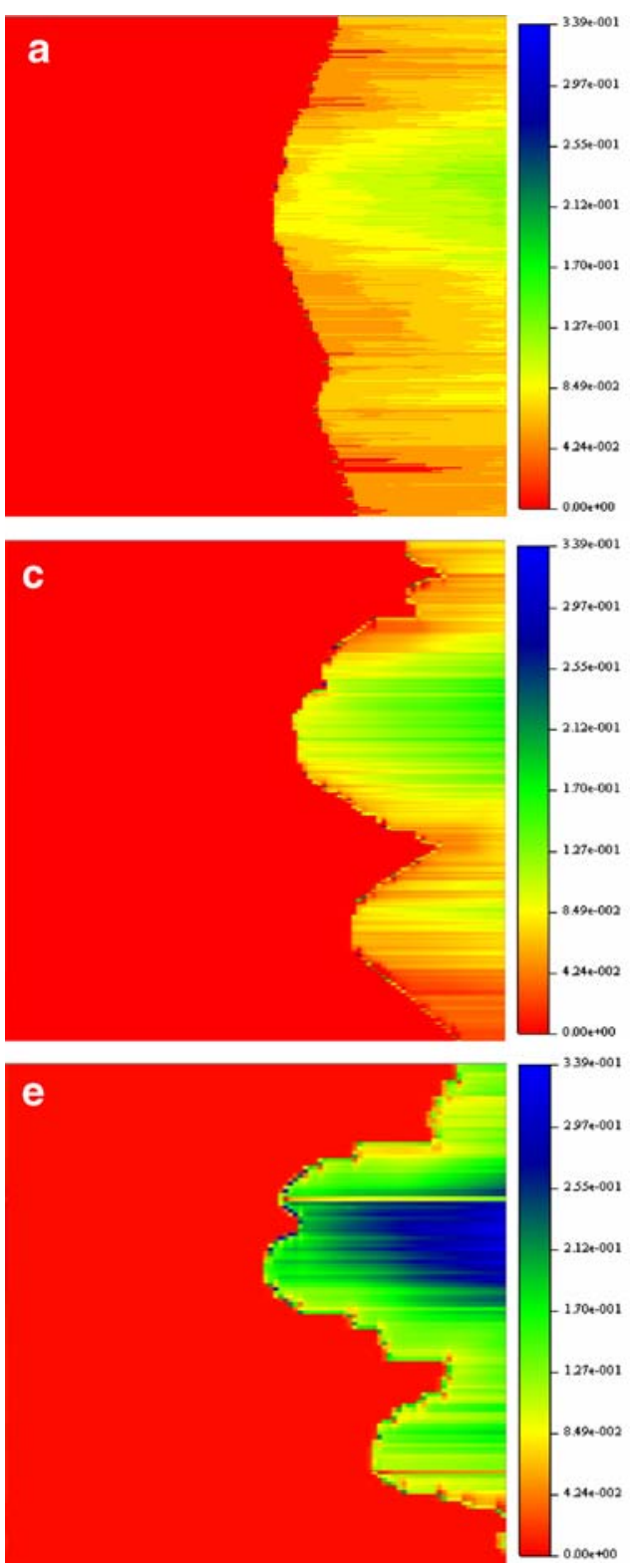
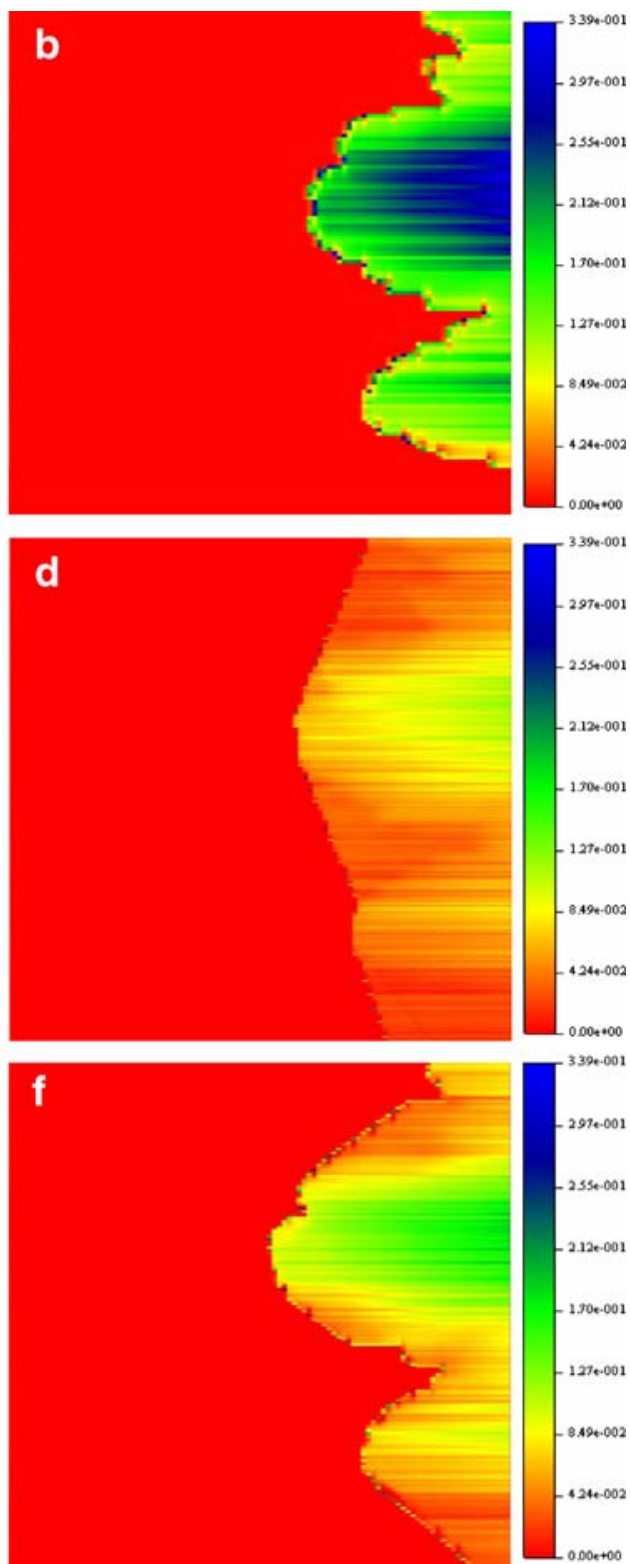

boundaries were not upscaled. The agreement between this case and the geological model, shown in Fig. 13a, is excellent. However, in contrast with Fig. 8 (for which $P_{\mathrm{c}}$ was neglected), all the other cases in which the blocks near the boundaries were upscaled at various levels could not reproduce the results obtained with the geological model, hence, demonstrating (a) the well-known end effect and (b) the accuracy of the upscaling method. These results also point out the significance of nonuniform upscaling. Uniform upscaling $\left(\varepsilon_{s}=\varepsilon_{d}=1.0\right)$ of the blocks (except perhaps those on the inlet and outlet faces) results in excessive averaging and smoothening of the heterogeneities (the permeabilities), hence, resulting in delayed breakthrough times. Therefore, the wavelet thresholds must be smaller than one. One the other hand, upscaling of the blocks at the system's inlet and outlet distorts the true relative significance of the capillary and viscous forces (recall that the capillary forces in the larger blocks are smaller). Thus, the optimal upscaling scenario, for the case in which the capillary pressure is significant, is one in which the blocks are coarsened nonuniformly (the wavelet thresholds $\varepsilon_{s}$ and $\varepsilon_{d}$ are smaller than unity), but those at the system's inlet and outlet are not upscaled. The same is true when there are injection and production wells in the system.

Figure 14 compares the distributions of $S_{\mathrm{w}}$ in the geological model and the optimal upscaled model (cor- 


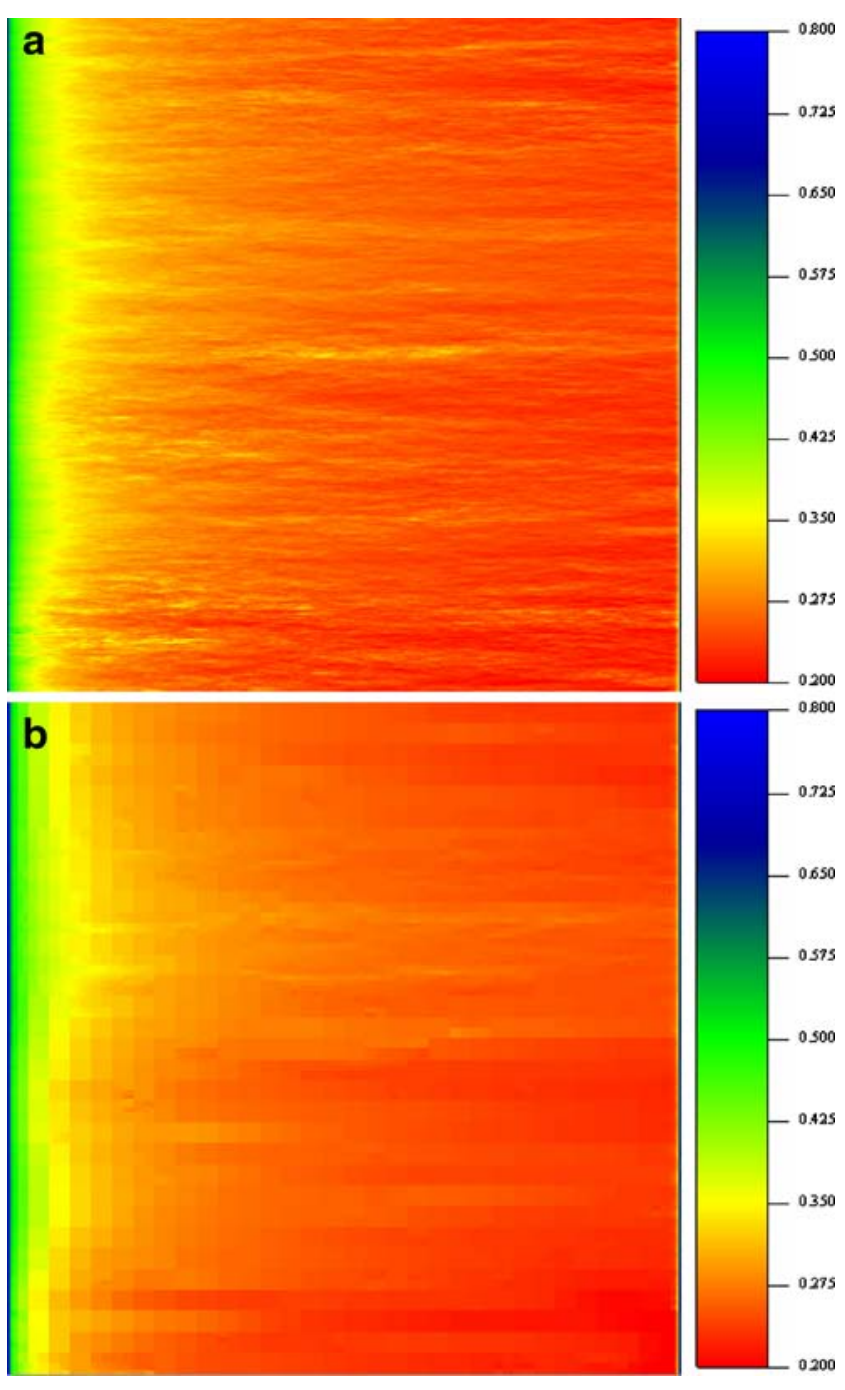

Fig. 14 a, b Same as in Fig. 9 but with the capillary pressure, given by Eq. 35, included. The results correspond to panel (d) of Fig. 13

responding to Fig. 13d) after 400 days. The capillary end-effect should give rise to large values of $S_{\mathrm{w}}$ at both ends, which has been captured accurately by the upscaled model. To make a more quantitative comparison between the geological and upscaled models, we present in Fig. 15 the computed time-dependence of the mean reservoir pressure, indicating very little difference between all the cases (the difference is at most $0.2 \%)$. At the same time, we should keep in mind that three levels of upscaling ( $l=3$ in Eqs. 17 and 18) greatly reduces the computation time (see Table 1). Figure 16 makes a comparison between the produced water flow rates in the geological and upscaled models, both with uniform $\left(\varepsilon_{s}=\varepsilon_{d}=1.0\right)$ and nonuniform upscaling and various levels $l$ of coarsening. As discussed above,

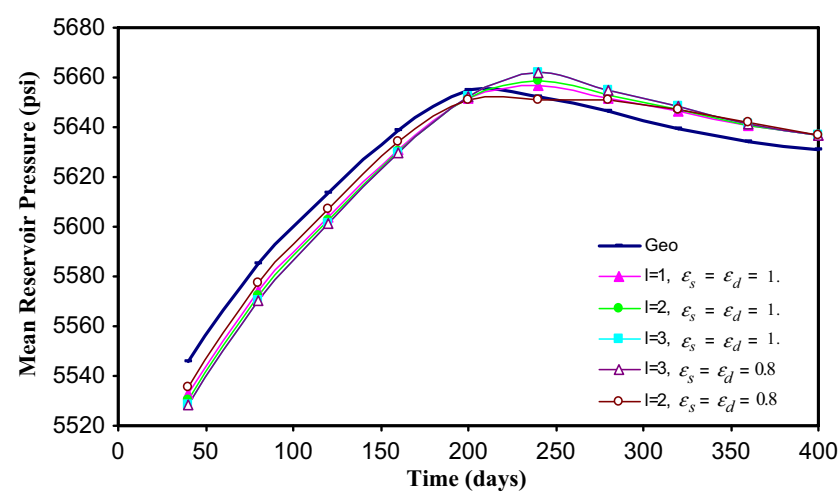

Fig. 15 Same as in Fig. 10 but with the capillary pressure, given by Eq. (3), included

nonuniform upscaling results in excellent agreement between the upscaled and geological models. Note that even one upscaling level reduces the total number of grid blocks from 262,144 in the geological model to about $10^{4}$ in the nonuniformly upscaled model, resulting in a speed-up factor of about 60.

Case 4 To test the upscaling scheme under more stringent conditions than those considered above, we carried out water-flooding simulations in a quarter of a nine-spot system. The permeability distribution was generated by FBM with a Hurst exponent, $H=0.2$, with $\left(K_{\min }, K_{\max }\right)=$ $(2 \mathrm{mD}, 1,400 \mathrm{mD})$, with a stratified structure, as shown in Fig. 17. The relative permeabilities were given by Eq. 34, while the capillary pressure was of the Corey type, given by

$$
P_{c}=10\left[\left(\frac{S_{\mathrm{w}}-S_{\mathrm{wc}}}{1-S_{\mathrm{wc}}-S_{\mathrm{or}}}\right)^{-1 / 5}-1\right],
$$

where $S_{\mathrm{wc}}$ is the connate water saturation. The porosity was assumed to be 0.3 . The initial pressure was $6,000 \mathrm{psi}$, and the initial water saturation was assumed to be 0.2 . Water was injected at one corner at a constant rate of $150 \mathrm{bbl} / \mathrm{day}$, the bottom-hole pressure at the producing wells was assumed to be 4,500 psi, and 300 days of the operations were simulated. The rest of the parameters were the same as those used in Case 1. After four and five levels of upscaling, the number of blocks decreased from the initial 262,144 to 5,632 and 3,073 , respectively. The resulting speed-up factors in the computations (see also below) were 958 and 6,203, respectively. This case is of particular interest, as there are three producing wells in the system (see Fig. 17), 
Table 1 Comparison of the computation times between those of the geological model (top row) and the upscaled grids and the resulting speed-up factors $(\mathrm{SF})$

\begin{tabular}{|c|c|c|c|c|c|c|}
\hline$\varepsilon$ & $l$ & Grid size & $t\left(P_{c}=0\right)$ & $\mathrm{SF}\left(P_{c}=0\right)$ & $t\left(P_{c} \neq 0\right)$ & $\mathrm{SF}\left(P_{c} \neq 0\right)$ \\
\hline & & 262,144 & $48.74 \mathrm{~h}$ & & $49.05 \mathrm{~h}$ & \\
\hline 1.0 & 3 & 1,600 & $98 \mathrm{~s}$ & 1,790 & $81 \mathrm{~s}$ & 2,180 \\
\hline 0.8 & 3 & 3,499 & $440 \mathrm{~s}$ & 398 & $334 \mathrm{~s}$ & 528 \\
\hline 1.0 & 2 & 2,368 & $270 \mathrm{~s}$ & 652 & $122 \mathrm{~s}$ & 1,447 \\
\hline 0.8 & 2 & 4,267 & $758 \mathrm{~s}$ & 231 & $408 \mathrm{~s}$ & 432 \\
\hline 1.0 & 1 & 3,904 & $867 \mathrm{~s}$ & 202 & $251 \mathrm{~s}$ & 703 \\
\hline
\end{tabular}

$\varepsilon=\varepsilon_{s}=\varepsilon_{d}$ refers to the wavelet thresholds, $l$ indicates the level of upscaling, while the grid size refers to the number of blocks in the grid

such that the flow of the fluids toward them can be parallel or perpendicular to the layers, or be at $45^{\circ}$ with respect to them.

Figure 18 compares the saturation distributions after 150 days, in both the geological and upscaled models, with the latter one obtained after four levels of upscaling. The agreement between the two is excellent. Figure 19 compares the water production rates at the three producing wells, computed with the geological
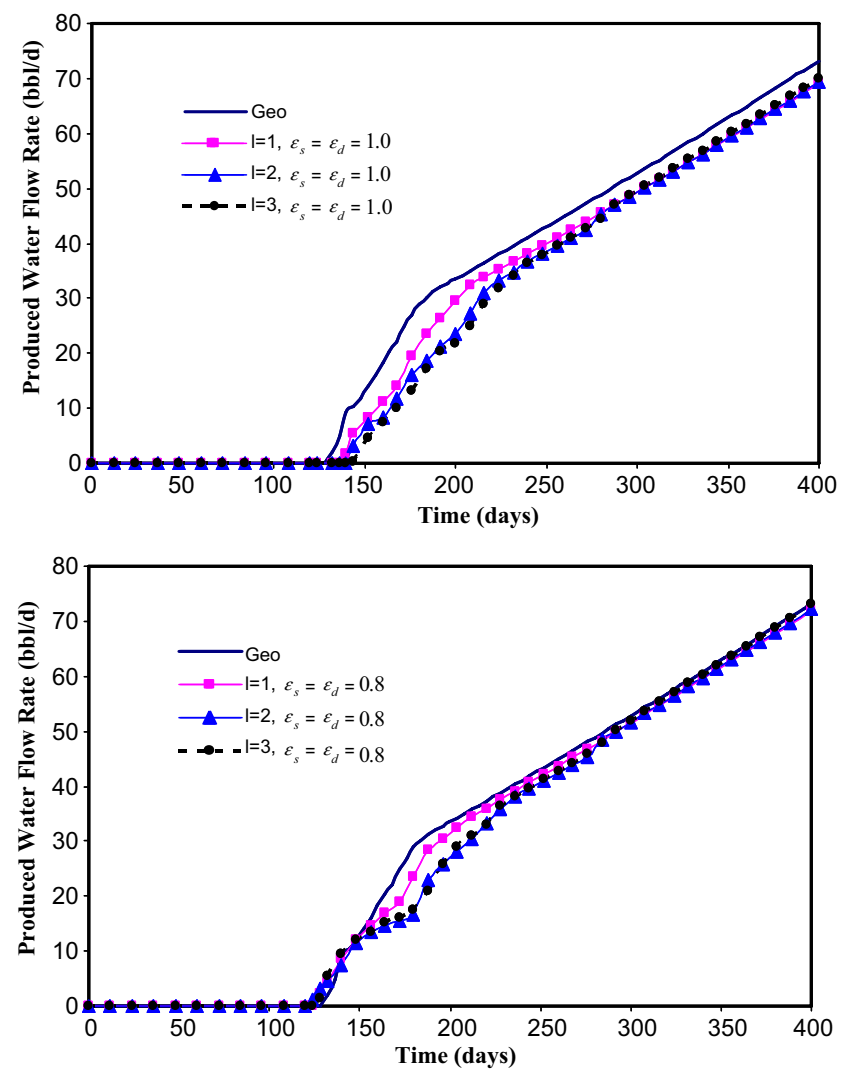

Fig. 16 Same as in Fig. 12 but with capillary pressure, given by Eq. (3), included model, and two upscaled grids obtained after four and five levels of upscaling. Once again, the agreement in all the cases is excellent. In particular, the breakthrough times are correctly predicted by the upscaled model. Figure 20 makes the same type of comparison that Fig. 19 does, but for the rates of oil production at the producing wells.

Case 5 As the final test of the upscaling method, we simulated water flooding in a $2 \mathrm{D}$ reservoir in which three intersecting fractures were inserted, with one of them being connected to the injection well. The three fractures were assumed to have a permeability of $20,000 \mathrm{mD}$ and to occupy one line of the grid. This is shown in Fig. 21. Note, in particular, the fine structure of the grid around the interface between the fractures and the matrix. The capillary pressure was ignored. Water was injected at one corner at a rate of $50 \mathrm{bbl} / \mathrm{day}$, and the fluids were extracted at the opposite corner. The rest of the parameters were the same as those of Case 4. Three levels of upscaling with $\varepsilon_{s}=\varepsilon_{d}=0.7,0.8$, and 0.9 were utilized which reduced the total number of grid blocks to $1,415,1,066$, and 775 , respectively.

The resulting speed-up factors in the computations (see also below) were, respectively, 269, 455, and 963. Figure 22 compares the saturation distributions after 10 days in the geological and the upscaled model after three upscaling levels. The agreement between the two is good. To make a more quantitative comparison between the two models, we compare in Fig. 23 the rate of oil production at the producing well in both the geological upscaled models. All the important features of the results in the geological model are produced correctly by the upscaled models. Moreover, as the level of upscaling increases, the agreement between 
Fig. 17 a Case 4 permeability distribution in the a geological model and $\mathbf{b}$ the upscaled grid after four levels of coarsening. One quarter of a nine-spot system is considered

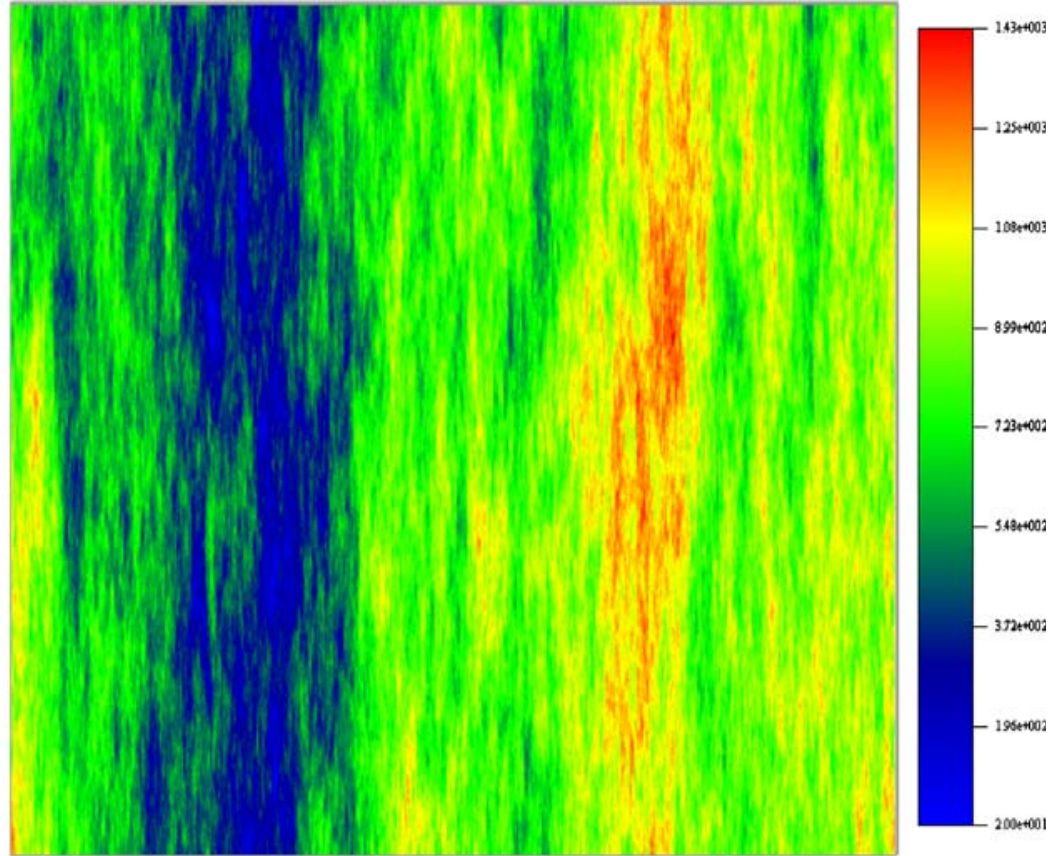

(a)

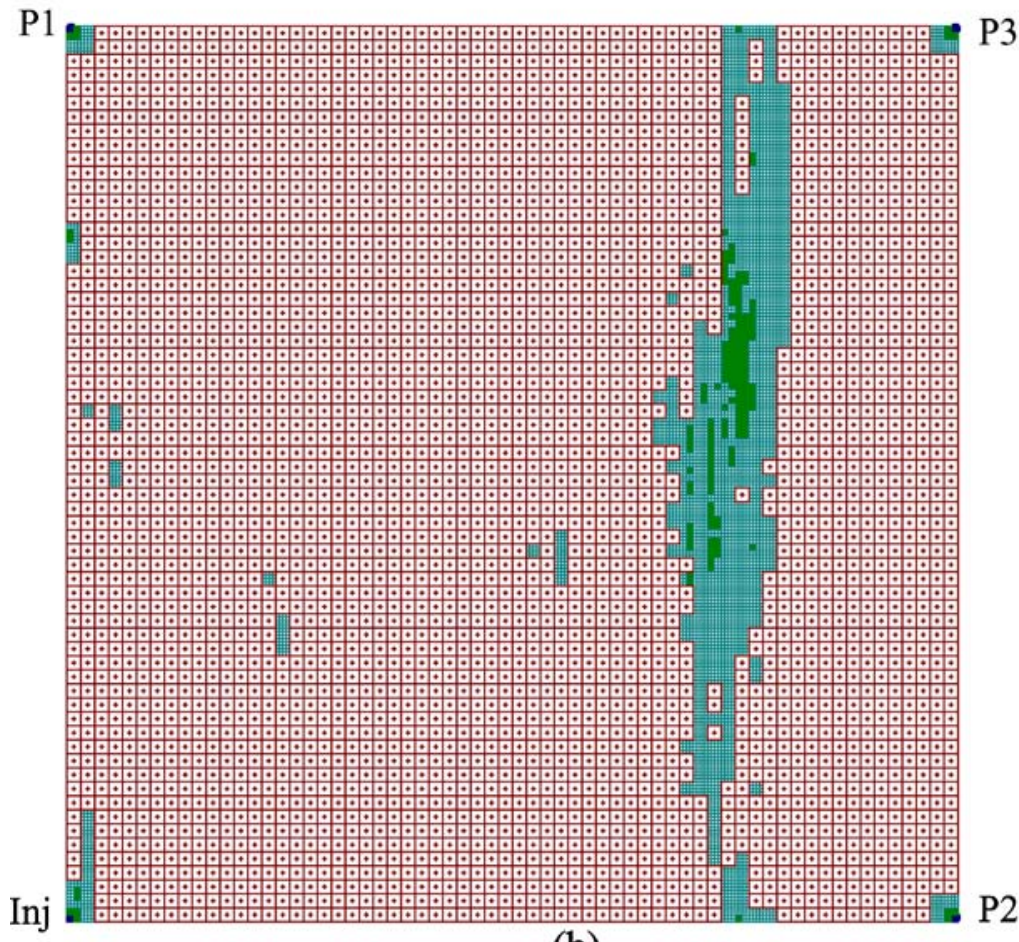

(b) the computed values in the two models also improves. Figure 24 compares the rates of water production at the producing well in the geological and upscaled models with the same level of agreement as that of Fig. 23.

\section{The speed-up of the wavelet upscaling}

In addition to its accuracy, any reasonable upscaling method must provide significant speed-up in the com- 


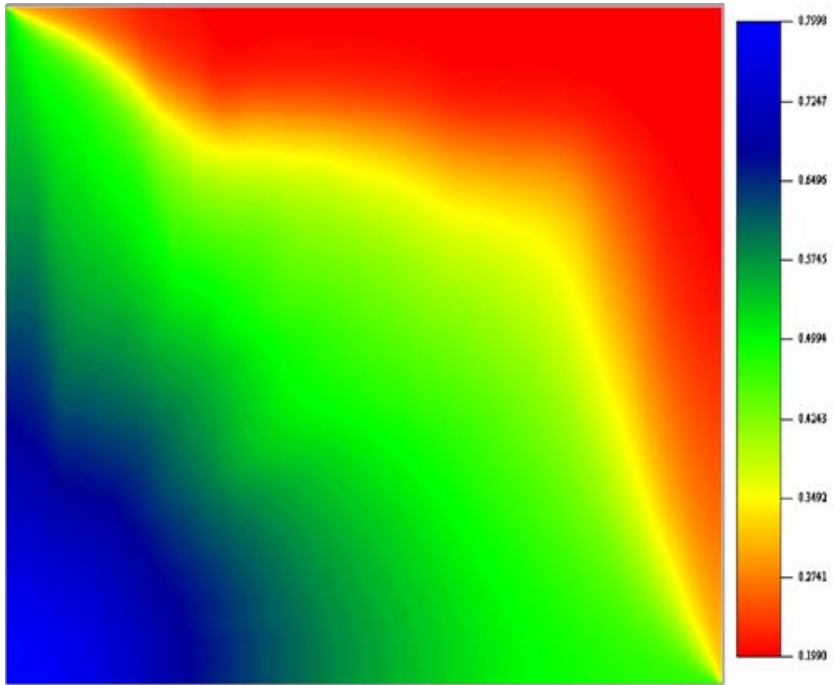

(a)

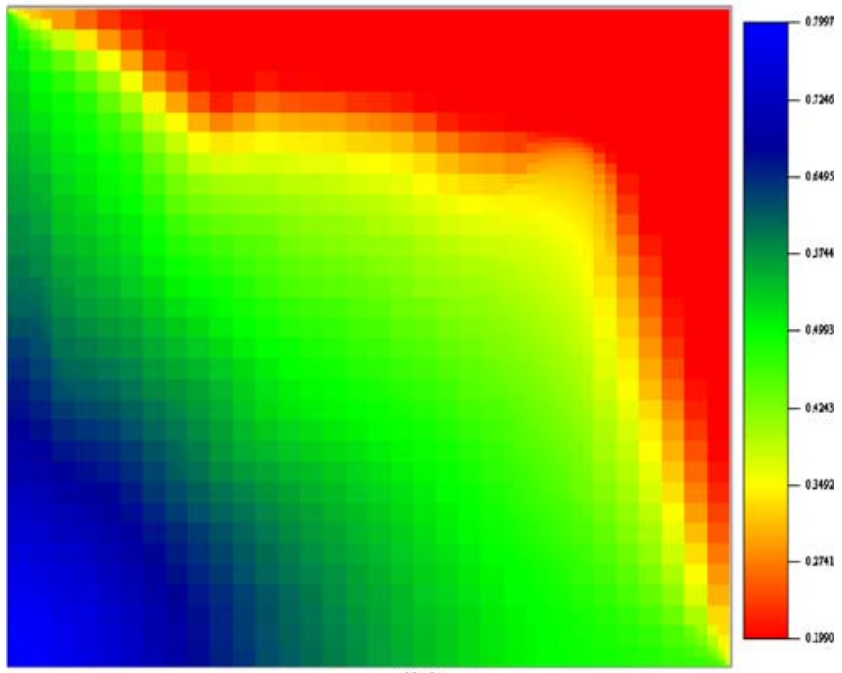

(b)

Fig. 18 Water saturation distribution in Case 4 reservoir (see Fig. 17) after 150 days in the a geological model and $\mathbf{b}$ upscaled model with four levels of upscaling

putations so that one can upscale a large geological model and obtain accurate results in a reasonable computation time. The upscaling method that we developed in this paper is highly efficient for the water-flooding problem in the quarter of a five-spot system and results in huge savings in the computation times. While the simulations with the geological model took about $475 \mathrm{~h}$ (19.8 days), those with the upscaled model took only $0.5 \mathrm{~h}$ (using the same computer), resulting in a speedup factor of 950. Using larger wavelet thresholds (than $\varepsilon_{s}=\varepsilon_{d}=0.8$ that we used) would result in even larger savings in the computation time.

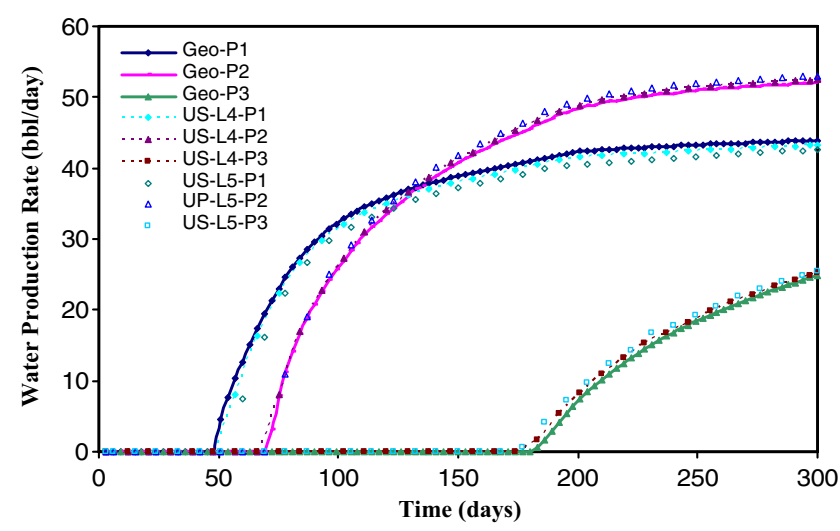

Fig. 19 Comparison of the water productions rates in Case 4 geological model ( $\mathrm{Geo}$ ) and the upscaled models with four and five levels of coarsening

The acceptable difference between the results obtained with the geological and upscaled models sets the desired values of the thresholds. In Table 1, we compare the CPU times for the first three cases (those for Cases 4 and 5 were already mentioned above) for water flooding in the systems in which water was injected at one face and oil and water were produced at the opposite face. The minimum speed-up factor that the wavelet-based yields is 202 but is as large as 6,203 (for Case 4, see above) - more than three orders of magnitude increase in the speed of the computations. We are not aware of any method that comes close to such great increases in the efficiency of the computations while, as the results presented above indicated, preserving the accuracy of the solutions.

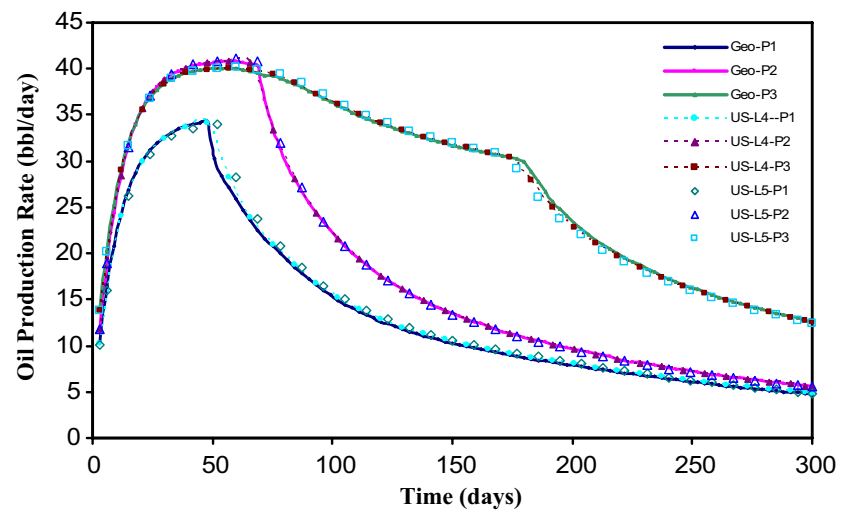

Fig. 20 Same as in Fig. 19 but comparing the oil production rates 
Fig. 21 a Case 5 permeability distribution with three fractures and $\mathbf{b}$ the resulting upscaled grid with $\varepsilon_{s}=\varepsilon_{d}=0.8$

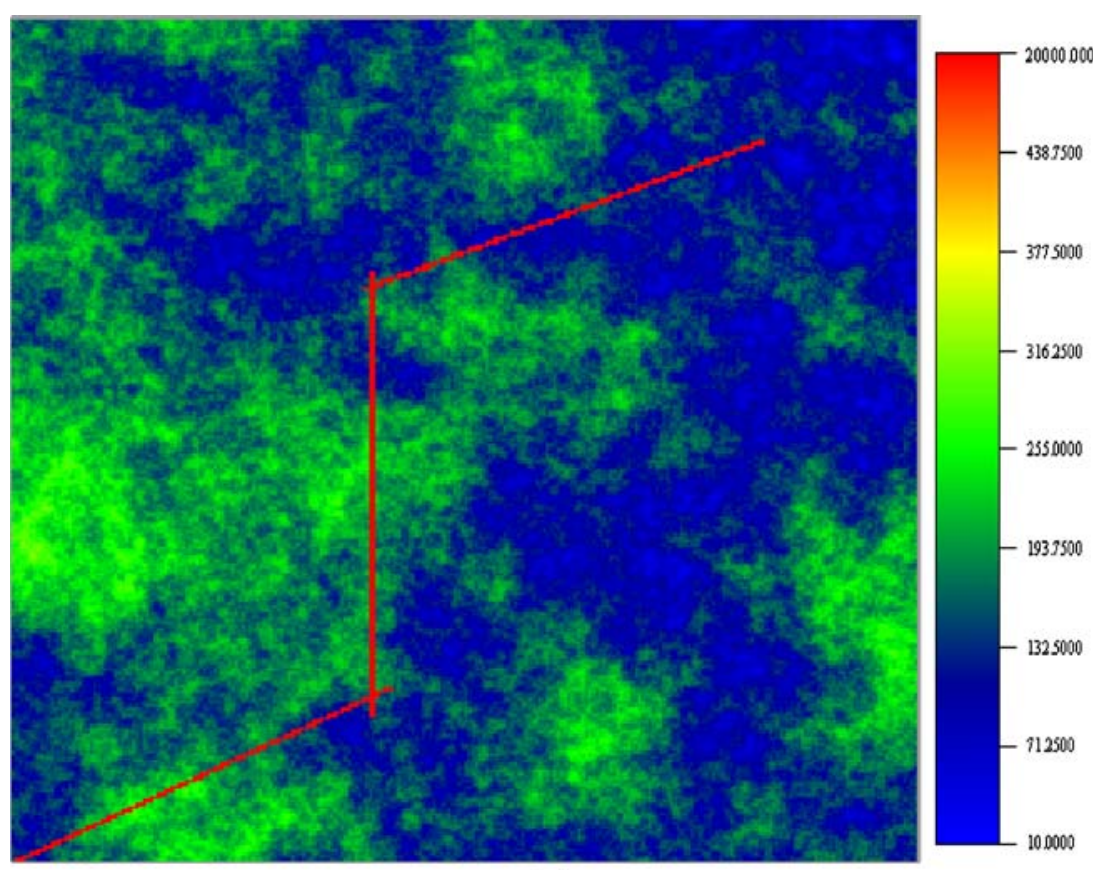

(a)

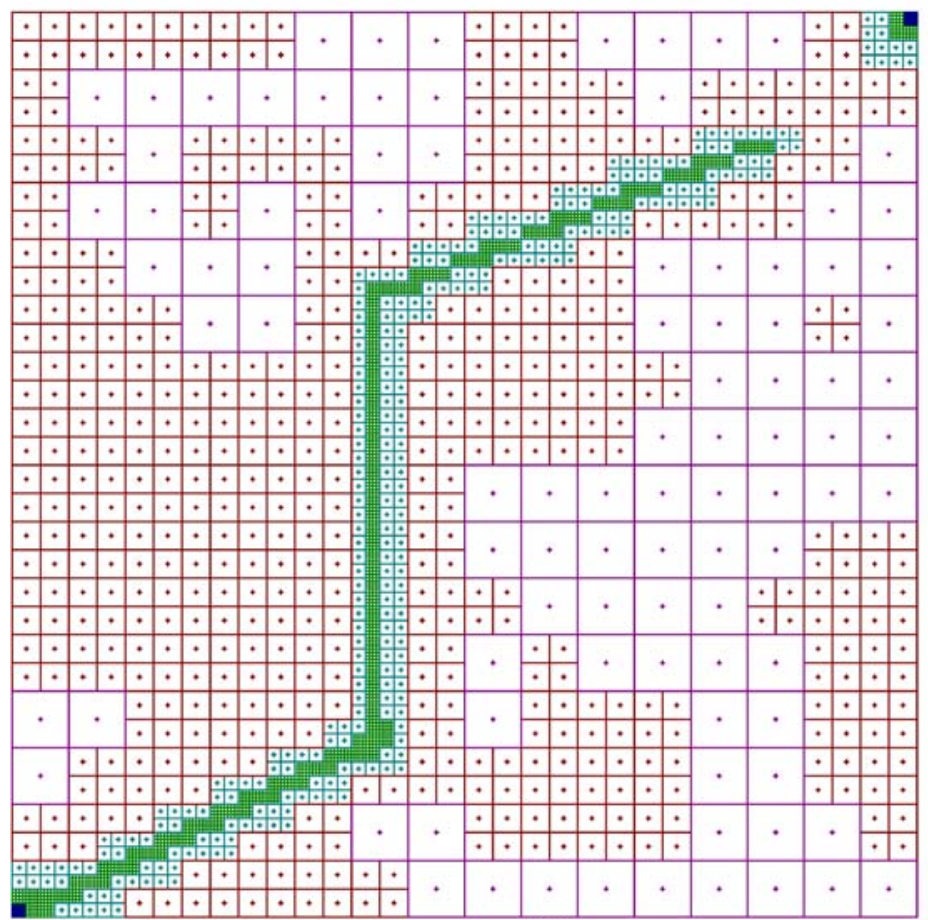

(b)
We should point out an important point regarding the efficiency of the wavelet method: the broader the permeability distribution, the more efficient is the wavelet method. The reason is clear: if the heterogeneities are distributed only relatively mildly, then significant fluid flow takes place through many sectors of the system. On the other hand, if the medium is highly heterogeneous, then only a small fraction of it contributes significantly 
Fig. 22 Water saturations after 10 days in a Case 5 geological model and $\mathbf{b}$ the upscaled model of Fig. 21
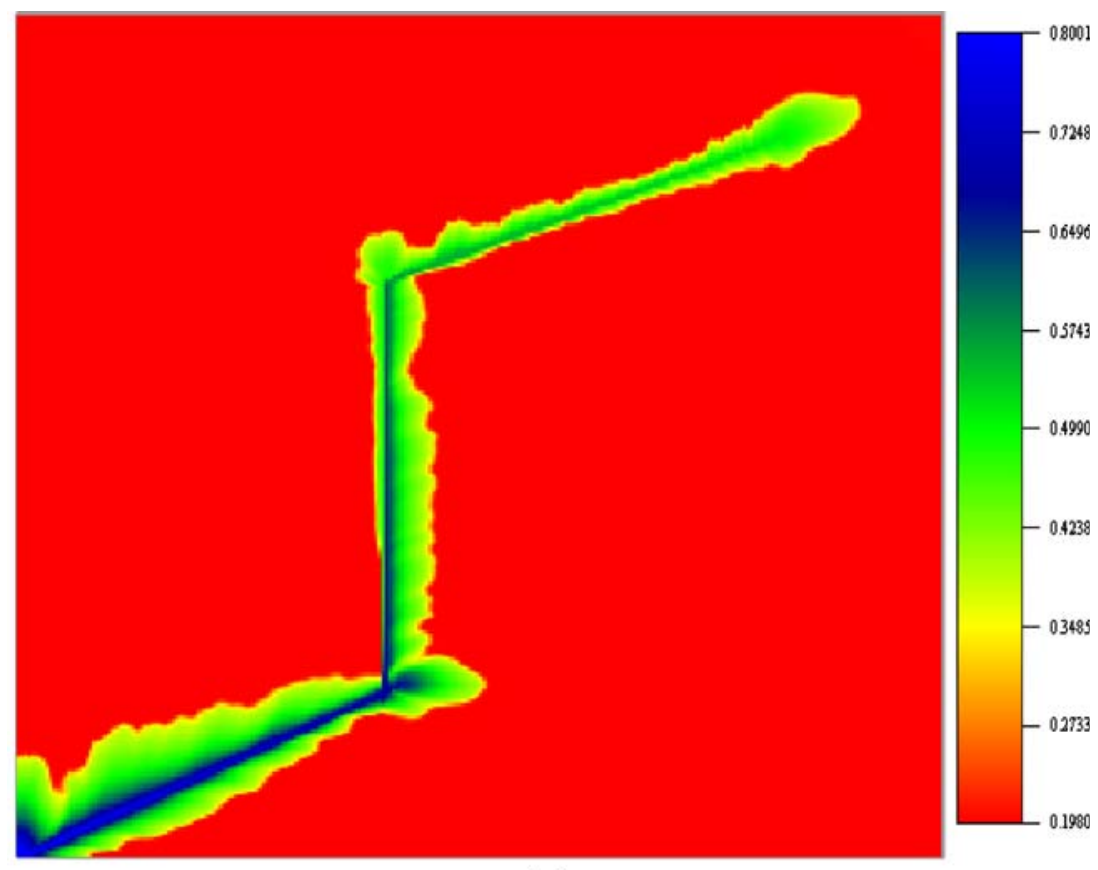

(a)
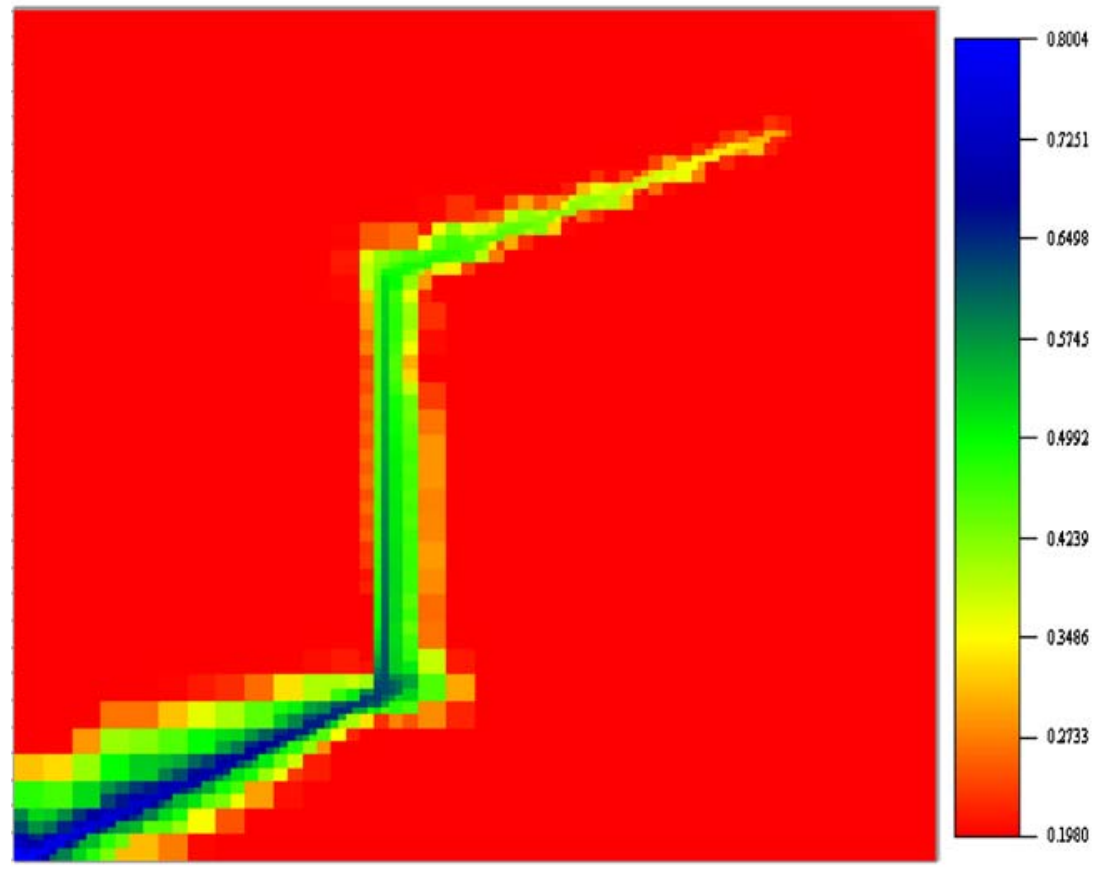

(b) to fluid flow. This important concept, known in physics of heterogeneous media as the critical-path concept, has proven to be very powerful for a wide variety of highly disordered media $[78,80]$. As a result, the number of grid blocks in the upscaled model, when the heterogeneities are mildly distributed, is larger than those in a highly heterogeneous medium.

This concept was recently demonstrated [67] for simulation of frequency-dependent conduction in a 3D heterogeneous material, in which the local conductivities 


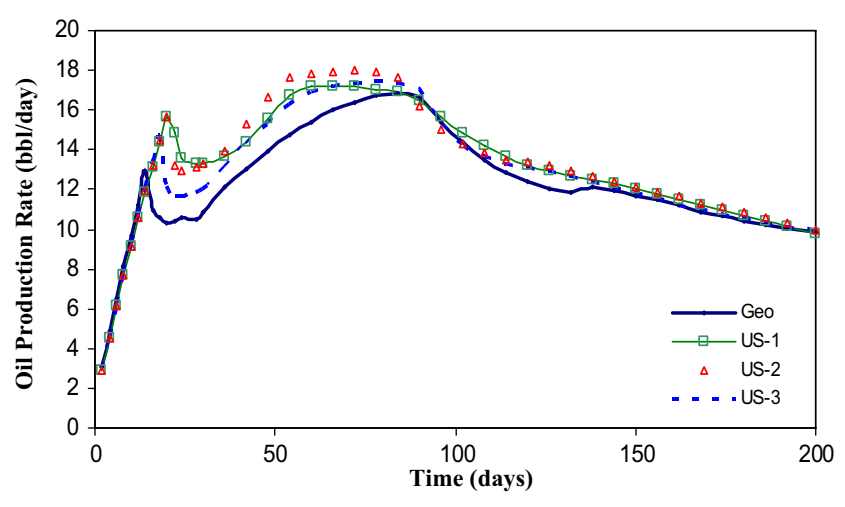

Fig. 23 Same as in Fig. 19 but for Case 5

were distributed over 12 orders of magnitude. The wavelet method reduced the number of grid blocks from 2,097,152 in the initial model to only 630. The difference between the effective conductivities of the two models, when the frequencies were varied over ten orders of magnitude, was at most 5\%. Elsewhere [73], we have demonstrated the same levels of efficiency and accuracy by simulating water flooding in the SPE-10 model [16], in which the permeabilities vary over eight orders of magnitude in a three-dimensional reservoir, exhibiting severe channeling, while the porosities vary over three orders of magnitude, with the fine-scale grid containing over 1.1 million grid blocks. The high accuracy of the results presented in the present paper and those for the SPE-10 model testify to the power of the method described in this paper. Further tests of the method, under a variety of conditions, are underway. The results will be reported elsewhere.

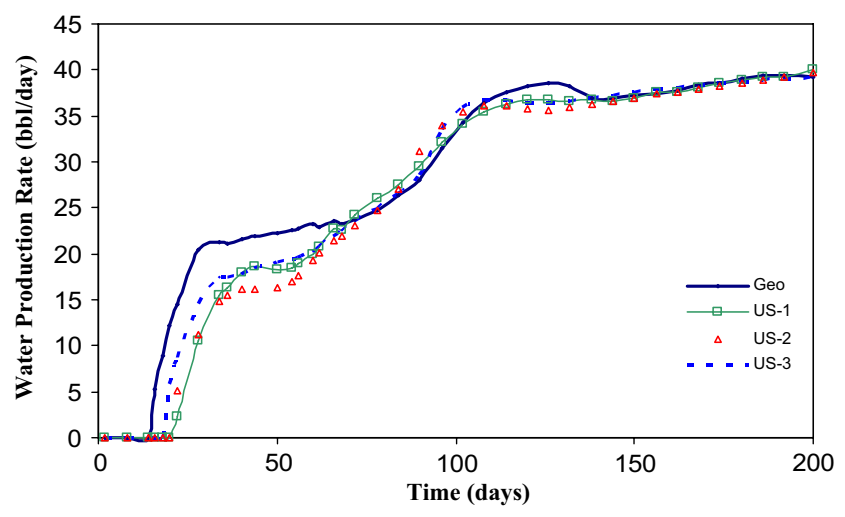

Fig. 24 Same as in Fig. 20 but for Case 5

\section{Summary}

We have developed an accurate and efficient method for upscaling of geological models of heterogeneous reservoirs for use in the simulation of two-phase fluid flow. The method is based solely on the wavelet transformation of the single-phase permeabilities; no two-phase flow property is upscaled. The technique, together with the improvements in the numerical methods that we suggested for solving the governing equations for two-phase flows, yields very significant speed-ups in the computations. In particular, a speedup factor of over 6,000 that we obtain for the Case 4 study in the presence of the capillary pressure is a demonstration of the method's high efficiency. Lohne et al. [54] proposed a two-stage upscaling method for two-phase flows from core to simulation scale, arguing that heterogeneities in the sub-geological scales (centimeter to meter scale) may have a significant effect on flow in a reservoir which cannot be captured if the upscaling starts at the geostatistical scale. They computed the effective properties for the geological units at the geostatistical scale by starting the upscaling at a small scale.

The effective properties at the reservoir simulation scale were obtained from a second upscaling using as the input the effective properties from the first step. Similar approaches were used earlier by $[18,88]$ in which the effect of very small-scale heterogeneities was included in the generation of the pseudo-functions for various geological facies. The wavelet-based method proposed here greatly reduces the computational cost of the two stages. This will be demonstrated in a future paper.

Finally, as Case 5 studied above hinted, the proposed method may be suited for development of upscaled models of fractured reservoirs, where one needs to use resolved grid blocks near the fractures in addition to the higher-permeability regions of the matrix and the nearwell zones. The results with this type of reservoirs will be reported in the near future.

Acknowledgements The work of M.R.R. was supported by the NIOC. We thank Drs. Fatemeh Ebrahimi, Amir Heidarinasab, and Manouchehr Haghighi for useful discussions.

Open Access This article is distributed under the terms of the Creative Commons Attribution Noncommercial License which permits any noncommercial use, distribution, and reproduction in any medium, provided the original author(s) and source are credited. 


\section{References}

1. Aavatsmark, I., Barkve, T., Mannseth, T.: Discretization on non-orthogonal, quadrilateral grids for inhomogeneous, anisotropic media. J. Comput. Phys. 127, 214 (1996)

2. Aavatsmark, I., Eigestad, G.T., Heimsund, B.-O., Mallison, B.T., Nordbotten, J.M., Øian, E.: A New Finite-volume Approach to Efficient Discretization on Challenging Grids, SPE 106435. SPE, Houston (2007)

3. Amaziane, B.: Global behavior of compressible three-phase flow in heterogeneous porous media. Trans. Porous Media 10, 43 (1993)

4. Arbogast, T., Bryant, S.L.: Numerical Subgrid Upscaling for Waterflood Simulations, SPE 66375. SPE, Houston (2001)

5. Athichanagorn, S., Horne, R.N., Kikani, J.: Processing and Interpretation of Long-term Data Acquired from Permanent Pressure Gages, SPE 56419. SPE, Houston (1999)

6. Audigane, P., Blunt, M.J.: Dual mesh method for upscaling in waterflood simulation. Trans. Porous Media 55, 71 (2004)

7. Barker, J.W., Dupouy P.: An analysis of dynamic pseudo relative permeabilities methods. In: Proceedings of the Fifth European Conference on the Mathematics of Oil Recovery, Leoben, Austria (1996)

8. Barker, J.W., Thibeau, S.: A Critical Review of the use of Pseudo Relative Permeabilities for Upscaling, SPE 35491. SPE, Houston (1996)

9. Beier, R.A.: Pseudo Relative Permeabilities from Fractal Distributions, SPE 24371. SPE, Houston (1992)

10. Bourgeat, A.: Homogenized behavior of two-phase flows in naturally fractured reservoirs with uniform fractures distribution. Comp. Meth. Appl. Mech. Eng. 47, 205 (1984)

11. Brewer, K.E., Wheatcraft, S.W.: Including Multi-scale Information in the Characterization of Hydraulic Conductivity Distributions. Wavelets in Geophysics, p. 213. Academic, San Diego (1994)

12. Castellini, A., Edwards M. G., Durlofsky, L.J.: Flow based modules for grid generation in two and three dimensions. In: Proceedings of Seventh European Conference on the Mathematics of Oil Recovery, Baveno, Italy (2000)

13. Chen, Y., Durlofsky, L.J.: Adaptive local-global upscaling for general flow scenarios in heterogeneous formations. Transp. Porous Media 62, 157 (2006)

14. Chen, Y., Durlofsky, L.J., Gerritsen, M.G., Wen, X.-H.: A coupled local-global upscaling approach for simulating flow in highly heterogeneous formations. Adv. Water Resour. 26, 1041 (2003)

15. Chen, Z., Hou, T.Y.: A mixed finite-element method for elliptic problems with rapidly oscillating coefficients. Math. Comput. 72, 541 (2002)

16. Christie, M.A., Blunt, M.J.: Tenth SPE Comparative Solution Project: a Comparison of Upscaling Techniques, SPE 66599. SPE, Houston (2001)

17. Chu, L., Schatzinger, R.A., Tham, M.K.: Application of Wavelet Analysis to Upscaling of Rock Properties, SPE 36517. SPE, Houston (1996)

18. Ciammetti, G., Ringrose, P.S., Good, T.R., Lewis, J.M.L., Sorbie, K.S.: Waterflood Recovery and Fluid Flow Upscaling in a Shallow Marian and Fluvial Sandstone Sequence, SPE 30783. SPE, Houston (1995)

19. Coats, K.H., Dempsey, J.R., Henderson, J.H.: The Use of Vertical Equilibrium in Two Dimensional Simulation of Three-dimensional Reservoir Performance, SPE 2797. SPE, Houston (1971)

20. Daubechies, I.: Orthonormal basis of compactly supported wavelets. Commun. Pure Appl. Math. 41, 901 (1988)
21. Daubechies, I.: Ten Lecture on Wavelets. SIAM, Philadelphia (1992)

22. Durlofsky, L.J.: Numerical calculation of equivalent grid block permeability tensors for heterogeneous porous media. Water Resour. Res. 27, 699 (1991)

23. Durlofsky, L.J., Jones, R.C., Milliken, W.J.: A non-uniform coarsening approach for the scale up of displacement processes in heterogeneous porous media. Adv. Water Resour. 20, 335 (1997)

24. Durlosfky, L.J., Milliken, W.J., Bemath, A.: Scaleup in the Near-well Region, SPE 61855. SPE, Houston (2000)

25. Ebrahimi, F.: Scale-up of Heterogeneous Porous Media and Flow and Transport therein Using Wavelet Transformation. Ph.D. Thesis, Ferdowsi University of Mashhad, Iran (2002)

26. Ebrahimi, F., Sahimi, M.: Multiresolution wavelet coarsening and analysis of transport in heterogeneous porous media. Physica. A 316, 160 (2002)

27. Ebrahimi, F., Sahimi, M.: Multiresolution wavelet scale up of unstable miscible displacements in flow through heterogeneous porous media. Transp. Porous Media 57, 75 (2004)

28. Ebrahimi, F., Sahimi, M.: Grid coarsening, simulation of transport processes in, and scale-up of heterogeneous media: application of multiresolution wavelet transformation. Mech. Mat. 38, 772 (2006)

29. Edwards, M.G.: Elimination of adaptive grid interface errors in the discrete cell centered pressure equation. J. Comput. Phys. 126, 356 (1996)

30. Edwards, M.G., Rogers, C.F.: Finite-volume discretization with imposed flux continuity for the general tensor pressure equation. Comput. Geosci. 2, 259 (1998)

31. Emmanuel, A.S., Cook, G.W.: Pseudo-relative Permeability for Well Modeling, SPE 4731. SPE, Houston (1974)

32. Forsyth, P.A., Sammon, P.H.: Local Mesh Refinement and Modeling of Faults and Pinchouts, SPE 13534. SPE, Houston (1985)

33. Gautier, Y., Blunt, M.J., Christie, M.A.: Nested gridding and streamline-based simulation for fast reservoir performance prediction. Comput. Geosci. 3, 295 (1999)

34. Gerritsen, M.G., Lambers, J.V.: Integration of local-global upscaling and grid adaptivity for simulation of subsurface flow. Comput. Geosci. 12, 193 (2008)

35. Harten, A.: On a class of highly accurate convective modeling procedure based on quadratic upstream interpolation. Comput. Methods Appl. Mech. Eng. 19, 59 (1979)

36. Harten, A., Osher, S.: Uniformly high-order accurate nonoscillatory schemes. SIAM. J. Numer. Anal. xx, 279 (1987)

37. Hearn, C.L.: Simulation of stratified waterflooding by pseudo relative permeability curves. JPT 23, 805 (1971)

38. Heidarinasab, A., Dabir, B., Sahimi, M.: Multiresolution wavelet-based simulation of transport and photochemical reactions in the atmosphere. Atmos. Envir. 38, 6381 (2004)

39. Hewett, T.A., Berhens, R.A.: Scaling laws in reservoir simulation and their use in a hybrid finite difference/streamtube approach to simulating the effects of permeability heterogeneity. In: Lake L.W. et al. (eds.) Reservoir Characterization II. Academic, San Diego (1991)

40. Holden, L., Lia, O.: A tensor estimator for the homogeneization of absolute permeability. Transp. Porous Media 8, 37 (1992)

41. Holden, L., Nielsen, B.F.: Global upscaling of permeability in heterogeneous reservoirs: the output least squares (OLS) method. Transp. Porous Media 40, 115 (2000)

42. Hou, T.Y., Wu, X.-H.: A multiscale finite-element method for elliptic problems in composite materials and porous media. J. Comput. Phys. 134, 169 (1997) 
43. Jacks, H.H., Smith, O.J.E., Mattax, C.C.: The Modeling of a Three-dimensional Reservoir by a Two-dimensional Reservoir Simulator-the Use of Dynamic Pseudo Functions, SPE 4071. SPE, Houston (1973)

44. Jenny, P., Wolfsteiner, C., Lee, S.H., Durlofsky, L.J.: Modeling Flow in Geometrically Complex Reservoirs Using Hexahedral Multi-block Grids, SPE 149. SPE, Houston (2002)

45. Jenny, P., Lee, S.H., Tchelepi, H.A.: Multi-scale finitevolume method for elliptic problems in subsurface flow simulations. J. Comput. Phys. 187, 47 (2003)

46. Jensen, J.L., Lake, L.W., Corbett, P.W.M., Goggin, D.J.: Statistics for Petroleum Engineers and Geoscientists. Elsevier, Amsterdam (2000)

47. Kikani, J., He, M.: Multiresolution Analysis of Long-term Pressure Transient Data using Wavelet Methods, SPE 48996. SPE, Houston (1998)

48. Kossack, C.A., Aasen, J.O., Opdal, S.T.: Scaling-up Laboratory Permeabilities and Rock Heterogeneities with Pseudo Functions for Field Simulations, SPE 18436. SPE, Houston (1989)

49. Kyte, J.R., Berry, D.W.: New Pseudo Functions to Control Numerical Dispersion, SPE 5105. SPE, Houston (1975)

50. Lambers, J.V., Gerritsen, M.G., Mallison, B.T.: Accurate local upscaling with variable compact multipoint transmissibility calculations. Comput. Geosci. 12, 399-416 (2008). doi:10.1007/s10596-007-9069-3

51. Lasseter, T.J., Waggoner, J.R., Lake, L.W.: Reservoir heterogeneities and their influence on ultimate recovery. In: Lake L.W. (ed.) Reservoir Characterization. Academic, Orlando (1986)

52. Lee, S.H., Durlofsky, L.J., Lough, M.F., Chen, W.H.: Finitedifference Simulation of Geologically Complex Reservoirs with Tensor Permeabilities, SPE 567. SPE, Houston (1998)

53. Lee, S.H., Tchelepi, H., Jenny, P., Dechant, L.: Implementation of a Flux-continuous Finite-difference Method for Stratigraphic, Hexahedron Grids, SPE 269. SPE, Houston (2002)

54. Lohne, A., Virnovsky, G., Durlofsky, L.J.: Two-stage Upscaling of Two-phase Flow: from Core to Simulation Scale, SPE 89422. SPE, Houston (2004)

55. Lu, P., Horne, R.N.: A Multiresolution Approach to Reservoir Parameter Estimation using Wavelet Analysis, SPE 62985. SPE, Houston (2000)

56. Lunati, I., Jenny, P.: Multiscale finite-volume method for compressible multiphase flow in porous media. J. Comput. Phys. 216, 616 (2006)

57. MacCarthy, J.F.: Comparison of fast algorithms for estimating large-scale permeabilities of heterogeneous media. Transp. Porous Media 19, 123 (1995)

58. Mallison, B., Gerritsen, M., Jessen, K., Orr, F.M.: High Order Upwind Schemes for Two-phase Multicomponent Flow, SPE 79671. SPE, Houston (2003)

59. Mehrabi, A.R., Rassamdana, H., Sahimi, M.: Characterization of long-range correlation in complex distributions and profiles. Phys. Rev. E 56, 712 (1997)

60. Mehrabi, A.R., Sahimi, M.: Coarsening of heterogeneous media: Application of wavelets. Phys. Rev. Lett. 79, 4385 (1997)

61. Molz, F.J., Rajaram, H., Lu, S.: Stochastic fractal-based models of heterogeneity in subsurface hydrology: origins, applications, limitations and future research questions. Rev. Geophys. 42, RG1002 (2004)

62. Moridis, G.J., Nikolaou, M., You, Y.: The Use of Wavelet Transforms in the Solution of Two-phase Flow Problems, SPE 169. SPE, Houston (1996)
63. Neupauer, R.M., Powell, K.L.: A fully-anisotropic Morlet wavelet to identify dominant orientations in a porous medium. Comput. Geosci. 31, 465 (2005)

64. Nielsen, B.F., Tveito, A.: An upscaling method for onephase flow in heterogeneous reservoirs. A weighted output least squares WOLS approach. Comput. Geosci. 2, 93 (1998)

65. Nordbotten, J.M., Aavatsmark, I., Eigestad, G.T.: Monotonicity of control volume methods. Numer. Math. 106, 255 (2006)

66. Panda, M.N., Mosher, C., Chopra, A.K.: Application of Wavelet Transforms to Reservoir Data Analysis and Scaling, SPE 3656. SPE, Houston (1996)

67. Pazhoohesh, E., Hamzehpour, H., Sahimi, M.: Numerical simulation of ac conduction in three-dimensional heterogeneous materials. Phys. Rev. B 73, 174206 (2006)

68. Peddibhotla, S., Gupta, A.D., Xue, G.: Multiphase Streamline Modeling in Three-dimensions: Further Generalizations and a Field Application, SPE 38003. SPE, Houston (1997)

69. Pickup, G.E., Ringrose, P.S., Jensen, J.L., Sorbie, K.S.: Permeability tensors for sedimentary structures. Math. Geol. 26, 227 (1994)

70. Pickup, G.E., Sorbie, K.S.: Development and Application of a New Two-phase Scaleup Method Based on Tensor Permeabilities, SPE 28586. SPE, Houston (1994)

71. Press, W.H., Teukolsky, S.A., Vetterling, W.T., Flannery, B.P.: Numerical Recipes, 2nd ed. Cambridge University Press, London (1992)

72. Quandalle, P., Besset, P.: Reduction of Grid Effects Due to Local Subgriding in Simulation using a Composite Grid, SPE 13527. SPE, Houston (1985)

73. Rasaei, M.R., Sahimi, M.: Upscaling and simulation of waterflooding in heterogeneous reservoirs using wavelet transformations: application to the SPE-10 model. Trans. Porous Media 72, 311 (2008)

74. Renard, P., de Marsily, G.: Calculating equivalent permeability, a review. Adv. Water Resour. 20, 253 (1997)

75. Roe, P.L.: Some contribution to the modeling of discontinuous flows. Lect. Appl. Math. 22, 163 (1985)

76. Rubin, B., Blunt, M.J.: Higher Order Implicit Flux Limiting Schemes for Black Oil Simulation, SPE 21222. SPE, Houston (1991)

77. Saez, A.E., Otero, C.J., Rusinek, I.: The effective homogeneous behavior of heterogeneous porous media. Trans. Porous Media 4, 213 (1989)

78. Sahimi, M.: Large-scale porous media and wavelet transformations. Comput. Sci. Eng. 5(4), 75 (2003a)

79. Sahimi, M.: Flow and Transport in Porous Media and Fractured Rock, 2nd Revised and Expanded edn. Wiley, Weinheim (2008)

80. Sahimi, M.: Heterogeneous Materials I \& II. Springer, New York (2003b)

81. Sahimi, M., Hashemi, M.: Wavelet identification of the spatial distribution of fractures. Geophys. Res. Lett. 28, 611 (2001)

82. Sahimi, M., Heidarinasab, A., Dabir, B.: Computer simulation of conduction in heterogeneous materials: application of wavelet transformations. Chem. Eng. Sci. 59, 4291 (2004)

83. Sahimi, M., Rasaei, M.R., Ebrahimi, F., Haghighi, M.: Upscaling of Unstable Displacements anmd Multiphase Flows using Multiresolution Wavelet Transformation, SPE 93320. SPE, Houston (2005)

84. Sammon, P.H., Kurihara, M., Liang, J.: Applying Highresolution Numerical Schemes in Reservoirs Described by Complex Corner-point Grids, SPE 66344. SPE, Houston (2001) 
85. Settari, A., Aziz, K.: Treatment of nonlinear terms in the numerical solution of partial differential solutions for multiphase flow in porous media. Int. J. Multiphase Flow 1, 817 (1975)

86. Shrivastava, V.K., Nghiem, L.X., Moore, R.G.: A Novel Approach for Incorporating Physical Dispersion in Miscible Displacement, SPE 77724. SPE, Houston (2002)

87. Soliman, M.Y., Ansah, J., Stephenson, S., Mandal, B.: Application of Wavelet Transform to Analysis of Pressure Transient Data, SPE 71571. SPE, Houston (2001)

88. Stephen, K.D., Clark, J.D., Pickup, G.E.: Modeling and Flow Simulations of a North Sea Turbidite Reservoir: Sensitivities and Upscaling, SPE 78292. SPE, Houston (2002)

89. Stone, H.L.: Rigorous Black Oil Pseudo Functions, SPE 21207. SPE, Houston (1991)

90. Straley, G.P.: A Material Balance Method for Driving Interblock Water/oil Pseudofunctions for Coarse Grid Reservoir Simulation, SPE 15621. SPE, Houston (1988)

91. Sweby, P.K.: High resolution TVD schemes using flux limiters. Lect. Appl. Math. 22, 289 (1985).

92. Thiele, M., Edwards, M.G.: Physically Based Higher Order Godunov Schemes for Compositional Simulation, SPE 66403. SPE, Houston (2001)

93. Thomas, C.W.: Principles of Hydrocarbon Reservoir Simulation. International Human Resources Development Corporation, Boston (1982)

94. van Leer, B.: Towards the ultimate conservative difference scheme, V. A second order sequal to Godunov's method. J. Comp. Phys. 32, 101 (1979)
95. Verdière, S., Guérillot, D., Thomas, J.M.: Dual mesh method for multiphase flows in heterogeneous reservoirs. In: Proceedings of Fifth European Conference on the Mathematics of Oil Recovery, Leoben, Austria (1996)

96. Verma, S., Aziz, K.: Two and three dimensional flexible grids for reservoir simulation. In: Proceedings of the Fifth European Conference on the Mathematics of Oil Recovery, Leoben, Austria (1996)

97. Wallstorm, T.C., Hou, S., Durlofsky, L.J.: Application of a New Two-phase Upscaling Technique to Realistic Reservoir Cross Sections, SPE 51939. SPE, Houston (1999)

98. Wattenbarger, R.C., Aziz, K., Orr, F.M.: High-throughput TVD-based Simulation of Tracer Flow, SPE 29097. SPE, Houston (1997)

99. Wen, X.-H., Gomez-Hernandez, J.J.: Upscaling hydraulic conductivities in heterogeneous media: an overview. J. Hydrol. 183, ix (1996)

100. Wen, X.-H., Durlofsky, L.J., Edwards, M.G.: Upscaling of channel systems in two dimensions using flow-based grids. Trans. Porous Media 51, 343 (2003)

101. White, C.D., Horne, R.N.: Computing Absolute Transmissibility in the Presence of Fine-scale Hetereogeneity, SPE 16011. SPE, Houston (1987)

102. Wolcott, D.S., Kazemi, H., Dean, R.H.: A Practical Method for Minimizing the Grid Orientation Effect in Reservoir Simulation, SPE 36723. SPE, Houston (1996)

103. Yee, H.C., Harten, A.: Implicit TVD schemes for hyperbolic conservative laws in curvilinear coordinates. AIAA J. 25, 266 (1987) 